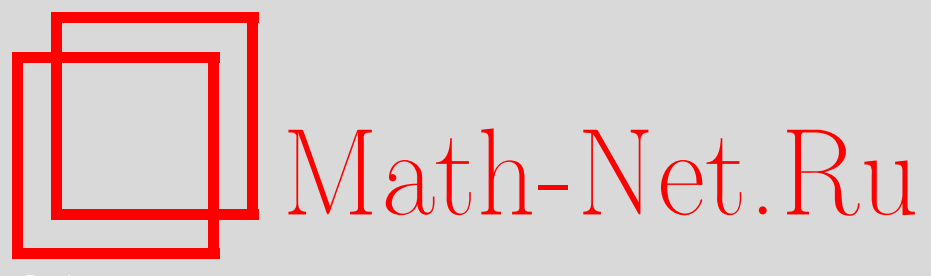

С. В. Бочкарев, Средние Валле Пуссена рядов Фурье для квадратичного спектра и спектров степенной плотности, УМН, 2014, том 69, выпуск 1, 125-162

DOI: https://doi.org/10.4213/rm9566

Использование Общероссийского математического портала Math-Net.Ru подразумевает, что вы прочитали и согласны с пользовательским соглашением http: //www. mathnet.ru/rus/agreement

Параметры загрузки:

IP : 54.237 .206 .68

26 апреля 2023 г., 13:13:09

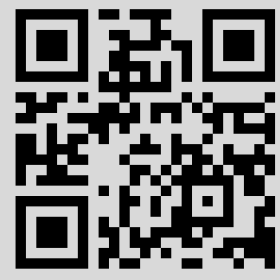




\title{
Средние Валле Пуссена рядов Фурье для квадратичного спектра и спектров степенной плотности
}

\begin{abstract}
С. В. Бочкарев
В статье предложен и развит новый метод исследования комплексных или вещественных тригонометрических рядов с различными спектрами. Метод основан на новых мультипликативных неравенствах, которые дают нижнюю оценку интегральной нормы средних Валле Пуссена и базируются на результатах, устанавливающих соответствующие аналоги теоремы Литтлвуда-Пэли в пространствах ВМО, Харди и Лоренца. Для спектров степенной плотности найдено зависящее от арифметических характеристик спектра и точное в предельных случаях описание класса модулей коэффициентов, для которых комплексные или вещественные тригонометрические ряды являются рядами Фурье. При этом для квадратичного спектра обобщены и усилены некоторые теоремы Харди и Литтлвуда, относящиеся к эллиптическим тэта-функциям. Установлены новые нижние оценки интегральной нормы экспоненциальных сумм для квадратичного спектра и степенных спектров с нецелыми показателями.

Библиография: 41 название.

Ключевые слова: ряды Фурье; средние Валле Пуссена; пространства ВМО, Харди и Лоренца; мультипликативные неравенства; спектры; экспоненциальные суммы; эллиптические функции и тэта-ряды; диофантовы уравнения.
\end{abstract}

DOI: $10.4213 / \mathrm{rm} 9566$

\section{СодЕРЖАниЕ}

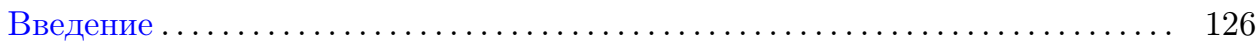

1. Тригонометрическая характеризация пространств ВМО и Харди . . . . 127

2. Мультипликативные нижние оценки $H^{1}$-нормы средних Валле Пуссена 129

3. Комплексные ряды Фурье со спектрами степенной плотности . . . . . . 133

4. Обобщенно-лакунарные спектры . . . . . . . . . . . . . . . 139

5. Квадратичный спектр, обобщение и усиление результатов Харди и Литтл-

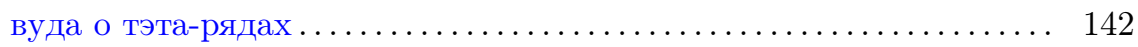

Работа выполнена при поддержке РФФИ (грант № 11-01-00417) и программ "Ведущие научные школы" (грант НШ-65-772-2010) и "Современные проблемы теоретической математики" Отделения математических наук РАН. 
6. Степенные спектры с нецелыми показателями, комплексный случай... 143

7. Мультипликативные неравенства для $L_{1}$-нормы вещественных тригонометрических рядов и полиномов ..................... 146

8. Вещественные тригонометрические ряды со спектрами степенной плот-

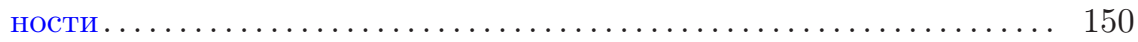

9. Квадратичный спектр и степенные спектры с нецелыми показателями, вещественный случай ............................ 156

Список литературы .................................... 159

\section{Введение}

Статья является продолжением работ автора [1]-[14], посвященных мультипликативным неравенствам для интегральной нормы и их приложениям.

Исследуемые неравенства дают мультипликативную нижнюю оценку $L_{1}$-нормы и включают в себя мультипликатор общего вида, который может быть как возрастающим, так и убывающим. Неравенства такого типа в мартингальном варианте (система Хаара) с возрастающим мультипликатором возникли при решении проблемы Зигмунда об абсолютной сходимости рядов Фурье функций ограниченной вариации в случае произвольной ограниченной полной ортонормированной системы. В контексте общего метода усреднений в теории ортогональных рядов здесь было произведено усреднение по специально сконструированной случайной сингулярной функции (см. [1], [3], [6], [15]).

Следует отметить, что другого подхода к этой проблеме Зигмунда до сих пор не найдено и в классическом случае тригонометрической системы. В частности, такой метод позволил установить логарифмический рост средних арифметических от функций Лебега ограниченных ортонормированных систем (см. [2], [4], [5]).

В последний период времени автор установил, что мультипликативные неравенства для $L_{1}$-нормы в тригонометрическом варианте при использовании убывающих мультипликаторов могут быть применены в гармоническом анализе для изучения комплексных и вещественных тригонометрических рядов с различными спектрами. Для доказательства таких неравенств требуются результаты, распространяющие теорию Литтлвуда-Пэли на предельные показатели $p=1$ и $p=\infty$. Заметим, что для функции $f \in L_{1}(0,2 \pi)$ сама двоичная функция Литтлвуда-Пэли может обращаться в бесконечность почти всюду. Поэтому, чтобы охватить предельные случаи, берется более гладкое разбиение единицы в образе Фурье. В этой работе применяется разложение, порожденное ядрами Валле Пуссена. Полные доказательства применяемых в данной статье результатов, относящихся к теории Литтлвуда-Пэли, содержатся в работе автора [7]. Подход, основанный на рядах Валле Пуссена, представляется естественным и достаточным для приложений в классическом случае единичного круга. Основным результатом здесь является характеризация пространства ВМО через разложение Валле Пуссена, из которой через теорему двойственности Феффермана и неравенство Хинчина непосредственно следует характеризация пространства Харди $H^{1}$ (см. [7]). 
Предложен и развит новый метод, который одновременно учитывает плотностные, а также теоретико-числовые и комбинаторные характеристики спектра и дает, в частности, точные результаты и для спектров с предельными плотностными характеристиками, и для спектров, обладающих предельными арифметическими свойствами (см. [8]-[14]).

В настоящей работе мультипликативные неравенства указанного типа установлены в наиболее общей форме, содержащей нижнюю оценку интегральной нормы средних Валле Пуссена рядов Фурье функций из $H^{1}$ и $L_{1}$, что позволяет как использовать их для оценки нормы в пространстве Харди, в частности для оценки $L_{1}$-нормы экспоненциальных сумм, так и применять их в вопросах теории рядов Фурье.

Для важного класса спектров степенной плотности, включая квадратичный спектр и степенные спектры с нецелыми показателями, решен вопрос о том, является ли рядом Фурье комплексный или вещественный тригонометрический ряд с заданными модулями коэффициентов.

При этом в классическом случае рядов по квадратам натуральных чисел усилены и обобщены результаты Харди и Литтлвуда, относящиеся к эллиптическим тэта-функциям и связанным с ними тригонометрическим рядам [16], [17].

Основные результаты этой статьи анонсированы в сообщении [14].

Обозначения: символы «и こозначают неравенство и эквивалентность с абсолютными постоянными, $C(\cdot)$ - константы, которые зависят от указанных параметров, а также могут зависеть от абсолютных постоянных, содержащихся в условиях, определяемых символами «и $\asymp, \log -$ натуральный логарифм, $|E|$ - мера множества $E$.

\section{1. Тригонометрическая характеризация пространств ВМО и Харди}

Пусть функция $f(z)$ регулярна в открытом единичном круге $|z|<1$,

$$
f(z)=\sum_{n=0}^{\infty} c_{n} z^{n},
$$

и $f(z)$ принадлежит пространству Харди $H^{1}$ (см. [18; т. 1, гл. VII, §7]). Тогда для почти всех $x \in[0,2 \pi)$ существует предел $\lim _{r \rightarrow 1} f\left(r e^{i x}\right)=f\left(e^{i x}\right)$ и выполняется равенство $\|f\|_{H^{1}}=\frac{1}{2 \pi} \int_{0}^{2 \pi}\left|f\left(e^{i x}\right)\right| d x$.

Через $\delta_{n}(x)$ обозначим двоичные пачки степенного ряда функции $f(z)$ при $r=1$ (см. (1)):

$$
\begin{gathered}
\delta_{0}(x)=c_{0}+c_{1} e^{i x} \\
\delta_{n}(x)=\sum_{k=2^{n-1}+1}^{2^{n}} c_{k} e^{i k x}, \quad n=1,2, \ldots
\end{gathered}
$$

Пусть $V_{n}(x)$ - ядра Валле Пуссена (см. [18; т. 1, гл. III, § 1, 2]). Обозначим $Q_{0}(x)=V_{1}(x)$ и положим

$$
Q_{n}(x)=V_{2^{n}}(x)-V_{2^{n-1}}(x), \quad n \geqslant 1 .
$$


Разложим $f\left(e^{i x}\right)$ в сходящийся почти всюду ряд Валле Пуссена (см. [19]):

$$
f\left(e^{i x}\right)=\sum_{n=0}^{\infty} \Delta_{n}(f, x),
$$

где $\Delta_{n}(f, x)=\frac{1}{\pi} \int_{0}^{2 \pi} f\left(e^{i u}\right) Q_{n}(x-u) d u$.

Частные суммы ряда (4) являются средними Валле Пуссена ряда Фурье степенного типа функции $f\left(e^{i x}\right)($ см. $(1),(3),(4))$ :

$$
\sigma_{2^{N}}(f, x):=\sigma_{2^{N}, 2^{N}}(f, x)=\sum_{n=0}^{N} \Delta_{n}(f, x) .
$$

Пространство Харди $H^{1}$ изоморфно вещественному пространству $H_{\mathrm{Re}}^{1}$ функций $f \in L_{1}(0,2 \pi)$ с нормой $\|f\|_{H_{\mathrm{Re}}^{1}}=\|f\|_{1}+\|\tilde{f}\|_{1}$, где $\tilde{f}-$ тригонометрически сопряженная функция. Как установил Ч. Фефферман [20], сопряженным пространством к $H_{\mathrm{Re}}^{1}$ является пространство BMO (см. [21; гл. VI, §1-4]).

Важную тригонометрическую характеризацию пространства ВМО дает следующая доказанная автором [7] теорема, которая является аналогом теоремы Литтлвуда-Пэли (см. [18; т. 2, гл. XV, § 2-4]) для показателя $p=\infty$.

Теорема 1. Для любой функиии $f \in L_{1}(0,2 \pi)$ имеет место соотношение

$$
\|f\|_{\mathrm{BMO}} \asymp \sup _{I}\left(\frac{1}{|I|} \int_{I} \sum_{2^{-n} \leqslant|I|} \Delta_{n}^{2}(f, x) d x\right)^{1 / 2},
$$

где $I$ - интервал.

Из теоремы 1 выводится характеризация пространства Харди через разложение Валле Пуссена для полидиска (см. [7], [11]). Пусть функция $f\left(z_{1}, \ldots, z_{m}\right)$, регулярная в открытом единичном $m$-мерном диске, принадлежит пространству Харди $H_{m}^{1}$, и пусть $f\left(e^{i x_{1}}, \ldots, e^{i x_{m}}\right)$ - ее граничное значение (см. [18; т. 2, гл. XVII, §4]).

Для $n=\left(n_{1}, \ldots, n_{m}\right)$ и $x=\left(x_{1}, \ldots, x_{m}\right)$ обозначим

$$
\begin{aligned}
\Delta_{n}(f, x)= & \frac{1}{\pi^{m}} \int_{0}^{2 \pi} \cdots \int_{0}^{2 \pi} f\left(e^{i u_{1}}, \ldots, e^{i u_{m}}\right) \\
& \times Q_{n_{1}}\left(x_{1}-u_{1}\right) \ldots Q_{n_{m}}\left(x_{m}-u_{m}\right) d u_{1} \ldots d u_{m} .
\end{aligned}
$$

Теорема 2. Если $f \in H_{m}^{1}$, то справедливы неравенства

$$
\|f\|_{H_{m}^{1}} \asymp\left\|\left(\sum_{n}\left|\Delta_{n}(f)\right|^{2}\right)^{1 / 2}\right\|_{L_{1}(0,2 \pi)^{m}} .
$$

ЗАмечАниЕ 1. Развитие теории Литтлвуда-Пэли происходило параллельно при взаимном влиянии с вопросами построения безусловных базисов и теорией вейвлетов, дающей конструктивное описание функциональных пространств. 
Так, в $L_{p}(1<p<\infty)$ безусловными базисами являются: система Хаара (Пэли, 1932), система Франклина (Бочкарев, 1974), ортогональные сплайны высших порядков (Чисельский, 1975). Существование безусловных базисов в $H^{1}$ установили Морей (1980), Карлесон (1980), Войташек (1982). Отметим также содержащийся в работах [22]-[24] метод построения полиномиальных, ортогональных безусловных базисов в конечномерных пространствах $H^{1}$, где использовался дискретный вариант системы Франклина в комбинации с интерполяцией посредством сдвигов комплексного ядра Дирихле.

ЗАмечАние 2. Характеризация (8) может быть выведена (с использованием неравенства Хинчина) из установленного в работе Койфмана и Вейса [25] утверждения типа теоремы Михлина-Хёрмандера о мультипликаторах, доказательство которого основано на атомарных и молекулярных представлениях для функции из $H^{1}$.

Доказательства теорем 1 и 2 сохраняют силу, если разложение (4) заменить на разложение $(m=1)$

$$
f\left(e^{i x}\right)=\sum_{n=0}^{\infty} \Delta_{n}^{*}(f, x),
$$

где

$$
\Delta_{n}^{*}(f, x)=\Delta_{2 n-1}(f, x)+\Delta_{2 n}(f, x), \quad n \geqslant 1 .
$$

Далее в статье будет использоваться разложение (9), (10).

\section{2. Мультипликативные нижние оценки $H^{1}$-нормы средних Валле Пуссена}

Следующая теорема дает мультипликативные нижние оценки средних Валле Пуссена функций из пространства Харди $H^{1}$ и включает в себя произвольный мультипликатор.

Она содержит предыдущие результаты в этом направлении (см. [8]-[12]) и позволяет определить, используя плотностные и арифметические свойства заданного спектра, какое условие на модули коэффициентов является необходимым для того, чтобы комплексный тригонометрический ряд по этому спектру являлся рядом Фурье.

Теорема 3. Пусть $f \in H^{1}, 2<p \leqslant \infty$, и пусть $\left\{\mu_{n}\right\}$ - последовательность неотрицательных чисел. Тогда при всех $N=1,2, \ldots$ выполняются следующие неравенства:

$$
\begin{aligned}
& \text { (I) }\left\|\sigma_{2^{2 N}}(f)\right\|_{1} \max _{0 \leqslant n \leqslant N}\left(\mu_{n} \sum_{q=0}^{n} \mu_{q}\left(\frac{\left\|\Delta_{q}^{*}\right\|_{p}^{p}}{\left\|\Delta_{q}^{*}\right\|_{2}^{2}}\right)^{2 /(p-2)}\right)^{1 / 2} \geqslant C(p) \sum_{n=0}^{N} \mu_{n}\left\|\Delta_{n}^{*}\right\|_{2}^{2} \\
& \text { (II) }\left\|\sigma_{2^{2 N}}(f)\right\|_{1} \max _{0 \leqslant n \leqslant N}\left(\mu_{n} \sum_{q=n}^{N} \mu_{q}\left(\frac{\left\|\Delta_{q}^{*}\right\|_{p}^{p}}{\left\|\Delta_{q}^{*}\right\|_{2}^{2}}\right)^{2 /(p-2)}\right)^{1 / 2} \geqslant C(p) \sum_{n=0}^{N} \mu_{n}\left\|\Delta_{n}^{*}\right\|_{2}^{2} .
\end{aligned}
$$


ДоказАтельство. (I) Будем считать, что $2<p<\infty$. Введем множества $E_{n} \subset[0,2 \pi), n=0,1,2, \ldots$ :

$$
E_{n}=\left\{\left|\Delta_{n}^{*}(f, x)\right| \leqslant \alpha(p)\left(\frac{\left\|\Delta_{n}^{*}\right\|_{p}^{p}}{\left\|\Delta_{n}^{*}\right\|_{2}^{2}}\right)^{1 /(p-2)}\right\}
$$

где $\alpha(p)$ - некоторая положительная постоянная.

Докажем, что постоянная $\alpha(p)$ может быть выбрана таким образом, что для множеств $C E_{n}=[0,2 \pi) \backslash E_{n}$ выполняется неравенство

$$
\int_{C E_{n}}\left|\Delta_{n}^{*}(f, x)\right|^{2} d x \leqslant \pi\left\|\Delta_{n}^{*}\right\|_{2}^{2}
$$

По неравенству Гёльдера

$$
\int_{C E_{n}}\left|\Delta_{n}^{*}(f, x)\right|^{2} d x \leqslant\left|C E_{n}\right|^{(p-2) / p}\left(\int_{C E_{n}}\left|\Delta_{n}^{*}(f, x)\right|^{p} d x\right)^{2 / p}
$$

Для оценки меры множества $C E_{n}$ применим неравенство Чебышёва. Имеем (см. (13))

$$
\begin{aligned}
\left|C E_{n}\right| & \leqslant(\alpha(p))^{-2}\left(\frac{\left\|\Delta_{n}^{*}\right\|_{p}^{p}}{\left\|\Delta_{n}^{*}\right\|_{2}^{2}}\right)^{2 /(2-p)} \int_{0}^{2 \pi}\left|\Delta_{n}^{*}(f, x)\right|^{2} d x \\
& =2 \pi(\alpha(p))^{-2}\left\|\Delta_{n}^{*}\right\|_{2}^{2 p /(p-2)}\left\|\Delta_{n}^{*}\right\|_{p}^{2 p /(2-p)} .
\end{aligned}
$$

Соединяя неравенства (15) и (16), получаем следующую оценку:

$$
\begin{gathered}
\int_{C E_{n}}\left|\Delta_{n}^{*}(f, x)\right|^{2} d x \leqslant(2 \pi)^{(p-2) / p}(\alpha(p))^{2(2-p) / p}\left\|\Delta_{n}^{*}\right\|_{2}^{2}\left\|\Delta_{n}^{*}\right\|_{p}^{-2} \\
\times\left(\int_{C E_{n}}\left|\Delta_{n}^{*}(f, x)\right|^{p} d x\right)^{2 / p} \leqslant 2 \pi(\alpha(p))^{2(2-p) / p}\left\|\Delta_{n}^{*}\right\|_{2}^{2} .
\end{gathered}
$$

Полагая теперь в определении (13)

$$
\alpha(p)=2^{p /(2(p-2))}
$$

и применяя оценку (17), находим, что выполняется неравенство (14). 
Таким образом (см. (14)), для любой последовательности неотрицательных чисел $\left\{\mu_{n}\right\}$ имеем

$$
\sum_{n=0}^{N} \mu_{n} \int_{E_{n}}\left|\Delta_{n}^{*}(f, x)\right|^{2} d x \geqslant \frac{1}{2} \sum_{n=0}^{N} \mu_{n} \int_{0}^{2 \pi}\left|\Delta_{n}^{*}(f, x)\right|^{2} d x
$$

Определим функции $g_{n}(x), n=0,1,2, \ldots, N$. Положим (см. (13))

$$
g_{n}(x)= \begin{cases}\left|\Delta_{n}^{*}(f, x)\right| & \text { при } x \in E_{n}, \\ 0 & \text { при } x \in C E_{n} .\end{cases}
$$

Ввиду (19), (20)

$$
\pi \sum_{n=0}^{N} \mu_{n}\left\|\Delta_{n}^{*}\right\|_{2}^{2} \leqslant \sum_{n=0}^{N} \mu_{n} \int_{0}^{2 \pi} g_{n}^{2}(x) d x
$$

Для любого $x \in[0,2 \pi)$ справедливо неравенство

$$
\begin{aligned}
\left(\sum_{n=0}^{N} \mu_{n} g_{n}^{2}(x)\right)^{2} & \leqslant 2 \sum_{n=0}^{N} \mu_{n} g_{n}^{2}(x) \sum_{m=0}^{n} \mu_{m} g_{m}^{2}(x) \\
& \leqslant 2 \max _{0 \leqslant q \leqslant N}\left(\mu_{q} \sum_{m=0}^{q} \mu_{m}\left\|g_{m}\right\|_{\infty}^{2}\right) \cdot \sum_{n=0}^{N} g_{n}^{2}(x)
\end{aligned}
$$

Применяя (22) и используя соотношения (20), (21), находим, что

$$
\begin{aligned}
\pi \sum_{n=0}^{N} \mu_{n}\left\|\Delta_{n}^{*}\right\|_{2}^{2} \leqslant & \sqrt{2} \max _{0 \leqslant q \leqslant N}\left(\mu_{q} \sum_{m=0}^{q} \mu_{m}\left\|g_{m}\right\|_{\infty}^{2}\right)^{1 / 2} \\
& \times \int_{0}^{2 \pi}\left(\sum_{n=0}^{N}\left|\Delta_{n}^{*}(f, x)\right|^{2}\right)^{1 / 2} d x .
\end{aligned}
$$

Так как согласно (13) и (20)

$$
\left\|g_{m}\right\|_{\infty}^{2} \leqslant(\alpha(p))^{2}\left(\frac{\left\|\Delta_{m}^{*}\right\|_{p}^{p}}{\left\|\Delta_{m}^{*}\right\|_{2}^{2}}\right)^{2 /(p-2)}
$$

то из (23) следует, что

$$
\begin{aligned}
\sum_{n=0}^{N} \mu_{n}\left\|\Delta_{n}^{*}\right\|_{2}^{2} \leqslant & \sqrt{2}(\alpha(p))^{2} \max _{0 \leqslant q \leqslant N}\left(\mu_{q} \sum_{m=0}^{q} \mu_{m}\left(\frac{\left\|\Delta_{m}^{*}\right\|_{p}^{p}}{\left\|\Delta_{m}^{*}\right\|_{2}^{2}}\right)^{2 /(p-2)}\right)^{1 / 2} \\
& \times \int_{0}^{2 \pi}\left(\sum_{n=0}^{N}\left|\Delta_{n}^{*}(f, x)\right|^{2}\right)^{1 / 2} d x
\end{aligned}
$$


Вместе с тем теорема 2, примененная к функции $\sigma_{2^{2 N}}(f)$ с разложением $(9)$, (10), дает оценку (см. (5), (8))

$$
\left\|\sigma_{2^{2 N}}(f)\right\|_{1} \geqslant C\left\|\left(\sum_{n=0}^{N}\left|\Delta_{n}^{*}(f)\right|^{2}\right)^{1 / 2}\right\|_{1} .
$$

Итак, окончательно получаем в силу соотношений (24) и (25), что справедливо неравенство (см. (18))

$$
\begin{gathered}
\left\|\sigma_{2^{2 N}}(f)\right\|_{1} \max _{0 \leqslant q \leqslant N}\left(\mu_{q} \sum_{m=0}^{q} \mu_{m}\left(\frac{\left\|\Delta_{m}^{*}\right\|_{p}^{p}}{\left\|\Delta_{m}^{*}\right\|_{2}^{2}}\right)^{2 /(p-2)}\right)^{1 / 2} \\
\geqslant C \cdot 2^{(3 p-2) /(2(2-p))} \sum_{n=0}^{N} \mu_{n}\left\|\Delta_{n}^{*}\right\|_{2}^{2}
\end{gathered}
$$

Тем самым (см. (11)) при $2<p<\infty$ пункт (I) теоремы 3 доказан. При $p=\infty$ доказательство проводится аналогично. Подобным образом устанавливается, что справедливо неравенство (12) пункта (II).

Поскольку средние Валле Пуссена сходятся к функции $f \in H^{1}$ по норме пространства Харди (см. [19]), то из теоремы 3 выводится теорема, содержащая нижние оценки $H^{1}$-нормы.

Теорема 4. Для любого $2<p \leqslant \infty$ и любой последовательности неотрицательных чисел $\mu_{n}$ выполняются следующие неравенства:

$$
\begin{aligned}
& \text { (I) }\|f\|_{H^{1}} \sup _{n}\left(\mu_{n} \sum_{q=0}^{n} \mu_{q}\left(\frac{\left\|\Delta_{q}^{*}\right\|_{p}^{p}}{\left\|\Delta_{q}^{*}\right\|_{2}^{2}}\right)^{2 /(p-2)}\right)^{1 / 2} \geqslant C(p) \sum_{n=0}^{\infty} \mu_{n}\left\|\Delta_{n}^{*}\right\|_{2}^{2} ; \\
& \text { (II) }\|f\|_{H^{1}} \sup _{n}\left(\mu_{n} \sum_{q=n}^{\infty} \mu_{q}\left(\frac{\left\|\Delta_{q}^{*}\right\|_{p}^{p}}{\left\|\Delta_{q}^{*}\right\|_{2}^{2}}\right)^{2 /(p-2)}\right)^{1 / 2} \geqslant C(p) \sum_{n=0}^{\infty} \mu_{n}\left\|\Delta_{n}^{*}\right\|_{2}^{2} .
\end{aligned}
$$

Если брать четные показатели $p$, то неравенства $(11),(12),(26),(27)$ дают возможность использовать арифметические свойства спектра функции $f \in H^{1}$. Наиболее важным для таких приложений является случай $p=4$. А при $p=\infty$ используются только плотностные свойства спектра.

ЗАмечАниЕ 3. Неравенства (12), (27) применяются с возрастающим мультипликатором $\left\{\mu_{n}\right\}$, что соответствует обобщенному дифференцированию, а неравенства (11), (26) - с убывающим $\left\{\mu_{n}\right\}$, который соответствует обобщенному интегрированию.

ЗАмечАниЕ 4. Вещественный вариант неравенства (27) с заменой $H^{1}$ на $L_{1}$ и возрастающим мультипликатором $\left\{\mu_{n}\right\}$ ранее был установлен автором для системы Хаара и применен в теории общих ортогональных рядов (см. [2]-[6]). Однако часть (I) теоремы 4 с убывающим мультипликатором в вещественном случае не имеет места (см. [7], [13]) ни для системы Хаара, ни для тригонометрической системы. 


\section{3. Комплексные ряды Фурье со спектрами степенной плотности}

Для последовательности натуральных чисел $1<m_{1}<m_{2}<\cdots<m_{n}<\cdots$ положим

$$
E_{q}=\left\{n: 2^{q-1}<m_{n} \leqslant 2^{q}\right\}, \quad q=1,2, \ldots, \quad \lambda_{q}=\operatorname{card} E_{q} .
$$

Арифметические свойства спектра $\left\{m_{n}\right\}$ характеризуются величинами $R(q)$, обозначающими число решений следующего уравнения (см. (28)):

$$
m_{n_{1}}+m_{n_{2}}=m_{n_{3}}+m_{n_{4}}, \quad n_{i} \in E_{q}, \quad i=1,2,3,4 .
$$

ОПРЕДЕЛЕНиЕ 1. Спектр $\left\{m_{n}\right\}$ имеет степенную плотность, если для некоторых постоянных

$$
1<A_{1} \leqslant 2 \quad \text { и } \quad A_{1} \leqslant A_{2}<\infty
$$

последовательность $\left\{\lambda_{q}\right\}$ удовлетворяет следующим соотношениям $\left(\lambda_{1}=1\right)$ :

$$
A_{1} \lambda_{q} \leqslant \lambda_{q+1} \leqslant A_{2} \lambda_{q}, \quad q=1,2, \ldots
$$

Пусть $\omega(n)$ - монотонная последовательность положительных чисел такая, что

$$
\omega\left(n^{2}\right) \asymp \omega(n), \quad n=2,3, \ldots ; \quad \omega(1)=\omega(2)=1 .
$$

Теорема 5. Пусть спектр $\left\{m_{n}\right\}$ имеет степенную плотность, а числа $R(q)$ удовлетворяют соотношению

$$
R(q) \ll \lambda_{q}^{\beta}\left(\log \lambda_{q}\right)^{\gamma}
$$

где $2 \leqslant \beta \leqslant 3, \gamma \leqslant 0$ при $\beta=3, \gamma \geqslant 0$ при $\beta=2$, и пусть функиия $f \in H^{1}$ представляется рядом

$$
f(z)=\sum_{n=1}^{\infty} c_{n} z^{m_{n}}, \quad|z|<1 .
$$

Тогда для любого $N=2,3, \ldots$ справедливы следующие неравенства.

(I) $E с л u$

$$
\left|c_{n}\right| \asymp n^{\rho} \omega(n) \quad \text { npu } 1 \leqslant n \leqslant N\left(1+A_{1}\right)^{2},
$$

mo

$$
\left\|\sigma_{m_{N}}(f)\right\|_{1} \geqslant\left\{\begin{array}{c}
C\left(A_{1}, A_{2}, \beta, \gamma, \rho\right) N^{\rho+(3-\beta) / 2} \omega(N)(\log N)^{-\gamma / 2} \\
n p u \beta \geqslant 2, \rho>\frac{1}{2}(\beta-3), \\
C\left(A_{1}, A_{2}, \beta, \gamma\right) \sum_{n=2}^{N} \frac{\omega(n)}{n(\log n)^{\gamma / 2}} \\
n p u \beta>2, \rho=\frac{1}{2}(\beta-3) .
\end{array}\right.
$$


(II) Если $\beta=2, \gamma>0$ и коэффичиенты степенного ряда (33) удовлетворяют соотношению

$$
\left|c_{n}\right| \asymp \frac{\omega(n)}{\sqrt{n}} \quad \text { npu } 1 \leqslant n \leqslant N\left(1+A_{1}\right)^{2},
$$

где для последовательности $\omega(n)$ выполняется условие

$$
\sum_{n=1}^{m} \frac{1}{\sqrt{n}} \omega\left(2^{n}\right) \ll \sqrt{m} \omega\left(2^{m}\right), \quad m=1,2, \ldots,
$$

то имеет место оценка

$$
\left\|\sigma_{m_{N}}(f)\right\|_{1} \gg C\left(A_{1}, A_{2}, \gamma\right) \sum_{n=2}^{N} \frac{\omega(n)}{n(\log n)^{(\gamma+1) / 2}} .
$$

ДокАзАтЕльство. Достаточно доказать теорему для случая, когда

$$
m_{N}=2^{2 M}
$$

при некотором $M=1,2, \ldots$. В общем случае, если $2^{2(M-1)}<m_{N}<2^{2 M}$, доказательство проводится аналогично. Вместо разложения (9), (10) следует взять разложение

$$
\sigma_{m_{N}}(f, x)=\sum_{n=0}^{M-2} \Delta_{n}^{*}(f, x)+\tilde{\Delta}_{M-1}^{*}(f, x),
$$

где

$$
\tilde{\Delta}_{M-1}^{*}(f, x)=\sigma_{m_{N}}(f, x)-\sum_{n=0}^{M-2} \Delta_{n}^{*}(f, x),
$$

для которого сохраняют силу основные результаты, справедливые для разложения (9), (10).

Обозначив через $\Lambda_{q}$ число точек спектра $m_{n}$, содержащихся в первых $q$ двоичных пачках,

$$
\Lambda_{q}=\sum_{k=1}^{q} \lambda_{k}
$$

и используя свойства спектров степенной плотности (см. (28), (30)), получаем, что справедливы соотношения

$$
\begin{gathered}
A_{1}^{q-1} \leqslant \lambda_{q} \leqslant 2^{q}, \\
\Lambda_{q} \leqslant \lambda_{q} \sum_{s=0}^{q-1} A_{1}^{-s} \leqslant \frac{A_{1}}{A_{1}-1} \lambda_{q} .
\end{gathered}
$$

Кроме того, из (39) и (40) следует, что

$$
\log N \ll M \ll C\left(A_{1}\right) \log N .
$$


Доказательство теоремы 5 основывается на неравенстве (11) из теоремы 3. Для того чтобы применить неравенство (11), нужно получить нижнюю оценку для $\left\|\Delta_{n}^{*}\right\|_{2}^{2}$ и верхнюю оценку для $\left\|\Delta_{n}^{*}\right\|_{4}^{4}$, а затем выбрать мультипликатор $\left\{\mu_{n}\right\}$.

По равенству Парсеваля, согласно соотношениям (2), (3), (9), (10), (28) и $(34)$, имеем $(1 \leqslant n \leqslant M)$

$$
\left\|\Delta_{n}^{*}\right\|_{2}^{2} \geqslant\left\|\delta_{2 n}\right\|_{2}^{2} \gg \sum_{s \in E_{2 n}}(\omega(s))^{2} s^{2 \rho} .
$$

Вместе с тем при $s \in E_{2 n}$ выполняются оценки

$$
\begin{gathered}
s \leqslant \Lambda_{2 n} \leqslant C\left(A_{1}\right) \lambda_{2 n}, \\
s>\Lambda_{2 n-1}>\lambda_{2 n-1} \geqslant \frac{1}{A_{2}} \lambda_{2 n} .
\end{gathered}
$$

Используя теперь соотношения (31), из (46) и (47) выводим, что при $s \in E_{2 n}$ справедливо неравенство (см. (42))

$$
C\left(A_{1}, A_{2}\right) \omega\left(2^{2 n}\right) \leqslant \omega(s) \leqslant C\left(A_{1}\right) \omega\left(2^{2 n}\right) .
$$

Соединяя оценки (45)-(48) и учитывая (28), получим

$$
\left\|\Delta_{n}^{*}\right\|_{2}^{2} \geqslant C\left(A_{1}, A_{2}, \rho\right) \lambda_{2 n}^{2 \rho+1}\left(\omega\left(2^{2 n}\right)\right)^{2} .
$$

Для получения верхней оценки $\left\|\Delta_{n}^{*}\right\|_{4}^{4}$ применим обобщенное неравенство Минковского. Имеем (см. (2), (3), (9), (10))

$$
\begin{aligned}
\left\|\Delta_{n}^{*}\right\|_{4}^{4} & \ll\left\|Q_{2 n-1}+Q_{2 n}\right\|_{1}^{4}\left\|\delta_{2 n-1}+\delta_{2 n}+\delta_{2 n+1}\right\|_{4}^{4} \\
& \ll\left\|\delta_{2 n-1}\right\|_{4}^{4}+\left\|\delta_{2 n}\right\|_{4}^{4}+\left\|\delta_{2 n+1}\right\|_{4}^{4} .
\end{aligned}
$$

Теперь оценим, например, предпоследнее слагаемое в правой части (50). Используя свойства системы экспонент $e^{i k x}$ и учитывая соотношения $(28),(29),(33)$, получим

$$
\left\|\delta_{2 n}\right\|_{4}^{4}=\sum_{\substack{m_{\nu_{1}}+m_{\nu_{2}}=m_{\nu_{3}}+m_{\nu_{4}} \\ \nu_{1}, \nu_{2}, \nu_{3}, \nu_{4} \in E_{2 n}}} c_{\nu_{1}} c_{\nu_{2}} \bar{c}_{\nu_{3}} \bar{c}_{\nu_{4}} \leqslant R(2 n) \max _{\nu \in E_{2 n}}\left|c_{\nu}\right|^{4}
$$

Отсюда (см. (50)), применяя (32), (34), а также используя (42), (46)-(48), заключаем, что при $1 \leqslant n \leqslant M$

$$
\left\|\Delta_{n}^{*}\right\|_{4}^{4} \leqslant C\left(A_{1}, A_{2}, \beta, \gamma, \rho\right) \lambda_{2 n}^{4 \rho+\beta}\left(\omega\left(2^{2 n}\right)\right)^{4} n^{\gamma} .
$$

Чтобы получить нижнюю оценку $L_{1}$-нормы средних Валле Пуссена для спектров степенной плотности, применим неравенство (11) из теоремы 3 с показателем $p=4$. Определим мультипликатор $\left\{\mu_{n}\right\}$ следующим образом:

$$
\mu_{n}=\lambda_{2 n}^{(1-\beta) / 2-\rho}\left(\omega\left(2^{2 n}\right)\right)^{-1} n^{-\gamma / 2}
$$


В силу неравенств (49) и (51) имеем

$$
\frac{\left\|\Delta_{n}^{*}\right\|_{4}^{4}}{\left\|\Delta_{n}^{*}\right\|_{2}^{2}} \leqslant C\left(A_{1}, A_{2}, \beta, \gamma, \rho\right) \lambda_{2 n}^{\beta+2 \rho-1}\left(\omega\left(2^{2 n}\right)\right)^{2} n^{\gamma} .
$$

Поэтому, используя (52), получим, что второй сомножитель в левой части неравенства (11) допускает оценку

$$
\begin{aligned}
\max _{n \leqslant M} & \left(\mu_{n} \sum_{q=1}^{n} \mu_{q} \frac{\left\|\Delta_{q}^{*}\right\|_{4}^{4}}{\left\|\Delta_{q}^{*}\right\|_{2}^{2}}\right) \leqslant C\left(A_{1}, A_{2}, \beta, \gamma, \rho\right) \\
& \times \max _{n \leqslant M}\left(\lambda_{2 n}^{(1-\beta) / 2-\rho}\left(\omega\left(2^{2 n}\right)\right)^{-1} n^{-\gamma / 2} \sum_{q=1}^{n} \lambda_{2 q}^{(\beta-1) / 2+\rho} \omega\left(2^{2 q}\right) q^{\gamma / 2}\right) .
\end{aligned}
$$

Рассмотрим показатель степени при $\lambda_{2 q}$ под знаком суммы в правой части (53). Неравенство $(\beta-1) / 2+\rho>0$ выполняется как в случае, если $\beta \geqslant 2$, $\rho>(\beta-3) / 2$, так и при $\beta>2, \rho \geqslant(\beta-3) / 2$. Таким образом, в случае пункта (I) теоремы 5 этот показатель всегда положителен и, следовательно, ввиду соотношений (30) и (31), выполняется неравенство

$$
\sum_{q=1}^{n} \lambda_{2 q}^{(\beta-1) / 2+\rho} \omega\left(2^{2 q}\right) q^{\gamma / 2} \leqslant C\left(A_{1}, A_{2}, \beta, \gamma, \rho\right) \lambda_{2 n}^{(\beta-1) / 2+\rho} \omega\left(2^{2 n}\right) n^{\gamma / 2} .
$$

Итак, имеем оценку (см. (53), (54))

$$
\max _{n \leqslant M}\left(\mu_{n} \sum_{q=1}^{n} \mu_{q} \frac{\left\|\Delta_{q}^{*}\right\|_{4}^{4}}{\left\|\Delta_{q}^{*}\right\|_{2}^{2}}\right) \leqslant C\left(A_{1}, A_{2}, \beta, \gamma, \rho\right) .
$$

Далее, согласно (49) и (52) сумма в правой части (11) удовлетворяет неравенству

$$
\sum_{n=1}^{M} \mu_{n}\left\|\Delta_{n}^{*}\right\|_{2}^{2} \geqslant C\left(A_{1}, A_{2}, \rho\right) \sum_{n=1}^{M} \lambda_{2 n}^{(3-\beta) / 2+\rho} \omega\left(2^{2 n}\right) n^{-\gamma / 2} .
$$

Рассмотрим случай $\rho>(\beta-3) / 2$. Тогда, используя $(30)$ и $(31)$, получим

$$
\sum_{n=1}^{M} \lambda_{2 n}^{(3-\beta) / 2+\rho} \omega\left(2^{2 n}\right) n^{-\gamma / 2} \leqslant C\left(A_{1}, A_{2}, \beta, \gamma, \rho\right) \lambda_{2 M}^{(3-\beta) / 2+\rho} \omega\left(2^{2 M}\right) M^{-\gamma / 2} .
$$

Поэтому, не ухудшая оценки, сумму в правой части неравенства (56) можно заменить последним слагаемым.

Следовательно (см. (56)), принимая во внимание соотношения (39), (44), получим при $\rho>(\beta-3) / 2$

$$
\sum_{n=1}^{M} \mu_{n}\left\|\Delta_{n}^{*}\right\|_{2}^{2} \geqslant C\left(A_{1}, A_{2}, \beta, \gamma, \rho\right) N^{\rho+(3-\beta) / 2} \omega(N)(\log N)^{-\gamma / 2},
$$

что вместе с оценкой (55) и доказывает справедливость пункта (I) теоремы 5 в этом случае (см. (11), (35), (55), (57)). 
Пусть теперь $\rho=(\beta-3) / 2$. Применяя оценки $(46)-(48)$ и учитывая $(28)$, имеем

$$
\sum_{n=1}^{M} \mu_{n}\left\|\Delta_{n}^{*}\right\|_{2}^{2} \geqslant C\left(A_{1}, A_{2}\right) \sum_{n=1}^{M} n^{-\gamma / 2} \omega\left(2^{2 n}\right) \geqslant C\left(A_{1}, A_{2}, \gamma\right) \sum_{n=1}^{2 M} n^{-\gamma / 2} \sum_{s \in E_{n}} \frac{\omega(s)}{s} .
$$

Но согласно (42) при $s \in E_{n}$

$$
s \geqslant \lambda_{n-1} \geqslant A_{1}^{n-2}
$$

и, значит (см. (28)),

$$
\log s \leqslant n \leqslant C\left(A_{1}\right) \log s .
$$

Соединяя (58) и (59), получим оценку

$$
\sum_{n=1}^{M} \mu_{n}\left\|\Delta_{n}^{*}\right\|_{2}^{2} \geqslant C\left(A_{1}, A_{2}, \gamma\right) \sum_{n=2}^{N} \frac{\omega(n)}{n(\log n)^{\gamma / 2}} .
$$

Таким образом (см. (35), (60)), пункт (I) теоремы 5 доказан.

(II) Пусть теперь $\beta=2, \rho=-1 / 2, \gamma \geqslant 0$. В этом случае определим мультипликатор $\left\{\mu_{n}\right\}$ соотношением

$$
\mu_{n}=\left(\omega\left(2^{2 n}\right)\right)^{-1} n^{-(\gamma+1) / 2} .
$$

Используя оценки (49) и (51), получим (см. (61))

$$
\begin{aligned}
\max _{n \leqslant M} & \left(\mu_{n} \sum_{q=1}^{n} \mu_{q} \frac{\left\|\Delta_{q}^{*}\right\|_{4}^{4}}{\left\|\Delta_{q}^{*}\right\|_{2}^{2}}\right) \leqslant C\left(A_{1}, A_{2}, \gamma\right) \\
& \times \max _{n \leqslant M}\left(\left(\omega\left(2^{2 n}\right)\right)^{-1} n^{-(\gamma+1) / 2} \sum_{q=1}^{n} \omega\left(2^{2 q}\right) q^{(\gamma-1) / 2}\right) .
\end{aligned}
$$

Но ввиду (37) при всех $\gamma \geqslant 0$ выполняется

$$
\sum_{q=1}^{n} \omega\left(2^{2 q}\right) q^{(\gamma-1) / 2} \ll \omega\left(2^{2 n}\right) n^{(\gamma+1) / 2}
$$

и, следовательно,

$$
\max _{n \leqslant M}\left(\mu_{n} \sum_{q=1}^{n} \mu_{q} \frac{\left\|\Delta_{q}^{*}\right\|_{4}^{4}}{\left\|\Delta_{q}^{*}\right\|_{2}^{2}}\right) \leqslant C\left(A_{1}, A_{2}, \gamma\right)
$$

Далее, имеем (см. (49), (61))

$$
\begin{aligned}
\sum_{n=1}^{M} \mu_{n}\left\|\Delta_{n}^{*}\right\|_{2}^{2} & \geqslant C\left(A_{1}, A_{2}\right) \sum_{n=1}^{M} n^{-(\gamma+1) / 2} \omega\left(2^{2 n}\right) \\
& \geqslant C\left(A_{1}, A_{2}, \gamma\right) \sum_{n=1}^{2 M} n^{-(\gamma+1) / 2} \sum_{s \in E_{n}} \frac{\omega(s)}{s}
\end{aligned}
$$


Отсюда получаем, учитывая (59), что

$$
\sum_{n=1}^{M} \mu_{n}\left\|\Delta_{n}^{*}\right\|_{2}^{2} \geqslant C\left(A_{1}, A_{2}, \gamma\right) \sum_{n=2}^{N} \frac{\omega(n)}{n(\log n)^{(\gamma+1) / 2}} .
$$

Таким образом, применяя неравенство (11), заключаем (см. (62), (64)), что справедливо неравенство

$$
\left\|\sigma_{m_{N}}(f)\right\|_{1} \geqslant C\left(A_{1}, A_{2}, \gamma\right) \sum_{n=2}^{N} \frac{\omega(n)}{n(\log n)^{(\gamma+1) / 2}} .
$$

Тем самым (см. (35), (38), (55), (57), (60)), теорема 5 полностью доказана.

Теорема 5 позволяет получить точное в определенном смысле описание класса модулей коэффициентов $\left\{c_{n}\right\}$, для которых соответствующие тригонометрические ряды являются рядами Фурье. Действительно, средние Валле Пуссена $\sigma_{N}(f)$ ряда Фурье функции $f \in H^{1}$ должны сходиться к $f$ по норме пространства $H^{1}$ (см. [19]), а неравенства (35), (38) дают возможность определить, при каких коэффициентах $\left\{c_{n}\right\}$ выполняется соотношение $\left\|\sigma_{N}(f)\right\|_{1} \rightarrow \infty$ при $N \rightarrow \infty$.

Таким образом, теорема 5 позволяет установить условия на модули коэффициентов комплексного тригонометрического ряда, необходимые для того, чтобы этот ряд был рядом Фурье. Вместе с тем условий на модули коэффициентов $\left\{c_{n}\right\}$, достаточных для того, чтобы тригонометрический ряд являлся рядом Фурье, и отличных от $\left\{c_{n}\right\} \in l_{2}$, не существует, так как если $\left\{c_{n}\right\} \notin l_{2}$, то случайная независимая расстановка знаков почти наверное дает не ряд Фурье (см. [18; гл. V, теорема 8.14]).

ТЕОРема 6. Пусть коэффициенты комплексного тригонометрического ряда

$$
\sum_{n=1}^{\infty} c_{n} e^{i m_{n} x}
$$

при всех $n=1,2, \ldots$ удовлетворяют соотношению (34), а арифметические свойства спектра $\left\{m_{n}\right\}$, имеющего степенную плотность (см. (30)), определяются соотношением (32). Тогда ряд (65) не являтся рядом Фуръе в следуюших случалх:

(I) при $\beta>2, \rho>(\beta-3) / 2$ или при $\rho=(\beta-3) / 2$, если выполняется соотношение

$$
\sum_{n=2}^{\infty} \frac{\omega(n)}{n(\log n)^{\gamma / 2}}=\infty
$$

(II) при $\beta=2, \gamma \geqslant 0, \rho>-1 / 2$ или при $\rho=-1 / 2$, если $\omega(n)$ удовлетворяет условию (37) и выполняется соотношение

$$
\sum_{n=2}^{\infty} \frac{\omega(n)}{n(\log n)^{(\gamma+1) / 2}}=\infty
$$


ЗАмечАниЕ 5 . При $\beta=3, \gamma=0, \rho=0$ соотношение (35) из теоремы 5 переходит в оценку, которую дает обобщенное неравенство Харди (см. [26], [27]), и, соответственно, теорема 6 дает здесь точное в случае линейного спектра необходимое условие (см. (66)):

$$
\sum_{n=1}^{\infty} \frac{\left|c_{n}\right|}{n}<\infty
$$

В остальных случаях $(2 \leqslant \beta<3, \rho \geqslant(\beta-3) / 2)$ теоремы 5 и 6 существенно усиливают результаты, которые следуют из обобщенного неравенства Харди.

Теоремы 5 и 6 точны и в другом предельном случае, когда условие (32) выполняется при $\beta=2, \gamma=0$. Однако такие спектры, которые естественно назвать обобщенно-лакунарными, целесообразно рассмотреть отдельно, так как здесь имеют место более общие утверждения.

\section{4. Обобщенно-лакунарные спектры}

ОПредЕлЕниЕ 2. Спектр $\left\{m_{n}\right\}$ назовем обобщенно-лакунарным, если числа $R(q)$, определяющие его арифметические свойства, удовлетворяют неравенству (см. (32))

$$
R(q) \ll \lambda_{q}^{2}, \quad q=1,2, \ldots .
$$

От других обобщений лакунарного по Адамару спектра (см. [28; т. 2, гл. 15]) это определение отличается тем, что используются только свойства спектра внутри двоичных пачек. Отметим, что вместо двоичных пачек здесь, как и в классической теории Литтлвуда-Пэли (см. [18; т. 2, гл. XV, §2-4]), можно взять любое другое разбиение, порожденное последовательностью $\left\{n_{k}\right\}$ такой, что $n_{k+1} / n_{k} \geqslant \alpha>1$ (см. [7]).

ТЕОРема 7. Пусть $\left\{m_{n}\right\}$ - обобщенно-лакунарный спектр (см. (68)), удовлетворяющий при некотором $A_{3} \geqslant 1$ условию

$$
A_{3}^{-1} \lambda_{q} \leqslant \lambda_{q+1} \leqslant A_{3} \lambda_{q}, \quad q=1,2, \ldots,
$$

и пусть для коэфбициентов $\left\{c_{n}\right\}$ при каждом $q=2,3, \ldots$ выполняется неравенство

$$
\left|c_{n_{2}}\right| \leqslant A_{4}\left|c_{n_{1}}\right|, \quad A_{4} \geqslant 1,
$$

где $n_{1}, n_{2} \in E_{q-1} \cup E_{q} \cup E_{q+1}$.

Тогда справедливы следующие утверждения.

(I) Если $f \in H^{1}$, то для любого $N=1,2, \ldots$ справедливо неравенство

$$
\left\|\sigma_{m_{N}}(f)\right\|_{1} \geqslant C\left(A_{3}, A_{4}\right)\left(\sum_{n=1}^{N}\left|c_{n}\right|^{2}\right)^{1 / 2} \text {. }
$$

(II) Если $\sum_{n=1}^{\infty}\left|c_{n}\right|^{2}=\infty$, то ряд

$$
\sum_{n=1}^{\infty} c_{n} e^{i m_{n} x}
$$

не является рядом Фуръе. 
ДокАЗАтельство. (I) Ограничимся случаем, когда $m_{N}=2^{2 M}$. В общей ситуации, если $2^{2(M-1)}<m_{N}<2^{2 M}$ при некотором $M=2,3, \ldots$, следует вместо разложения (9), (10) взять разложение (40), (41) и далее доказательство проводится аналогично.

В силу теоремы 3 , полагая в неравенстве (11) мультипликатор $\mu_{n}$ равным 1 , $n=1,2, \ldots$, получаем следующее неравенство $(p=4)$ :

$$
\left\|\sigma_{2^{2 M}}(f)\right\|_{1} \gg\left(\sum_{n=1}^{M} \frac{\left\|\Delta_{n}^{*}\right\|_{4}^{4}}{\left\|\Delta_{n}^{*}\right\|_{2}^{2}}\right)^{-1 / 2} \sum_{n=1}^{M}\left\|\Delta_{n}^{*}\right\|_{2}^{2}
$$

Применяя обобщенное неравенство Минковского, имеем (см. (50))

$$
\left\|\Delta_{n}^{*}\right\|_{4}^{4} \ll\left\|\delta_{2 n-1}\right\|_{4}^{4}+\left\|\delta_{2 n}\right\|_{4}^{4}+\left\|\delta_{2 n+1}\right\|_{4}^{4}
$$

Докажем, что для каждого $n=1,2, \ldots$ при любом $q=2 n-1,2 n, 2 n+1$ выполняется неравенство

$$
\frac{\left\|\delta_{q}\right\|_{4}^{4}}{\left\|\delta_{2 n}\right\|_{2}^{2}} \leqslant C\left(A_{3}, A_{4}\right) \sum_{k \in E_{2 n}}\left|c_{k}\right|^{2}
$$

Действительно, используя соотношения (28), (29), (69), (70), получим

$$
\begin{aligned}
\left\|\delta_{q}\right\|_{4}^{4} & =\frac{1}{2 \pi} \int_{0}^{2 \pi}\left|\sum_{\nu \in E_{q}} c_{\nu} e^{i m_{\nu} x}\right|^{4} d x \\
& =\sum_{\substack{m_{\nu_{1}}+m_{\nu_{2}}=m_{\nu_{3}} \in E_{q}, m_{\nu_{4}} \\
j=1,2,3,4}} c_{\nu_{1}} c_{\nu_{2}} \bar{c}_{\nu_{3}} \bar{c}_{\nu_{4}} \leqslant R(q) \max _{\nu \in E_{q}}\left|c_{\nu}\right|^{4} \\
& \leqslant A_{4}^{4} \lambda_{q}^{2} \min _{\nu \in E_{2 n}}\left|c_{\nu}\right|^{4} \leqslant A_{3}^{2} A_{4}^{4} \lambda_{2 n}^{2} \min _{\nu \in E_{2 n}}\left|c_{\nu}\right|^{4} \leqslant A_{3}^{2} A_{4}^{4}\left(\sum_{\nu \in E_{2 n}}\left|c_{\nu}\right|^{2}\right)^{2}
\end{aligned}
$$

А так как (см. (28)) $\left\|\delta_{2 n}\right\|_{2}^{2}=\sum_{\nu \in E_{2 n}}\left|c_{\nu}\right|^{2}$, то (73) следует из (74). Теперь, используя (73) и учитывая, что (см. (10))

$$
\left\|\Delta_{n}^{*}\right\|_{2}^{2} \geqslant\left\|\delta_{2 n}\right\|_{2}^{2}
$$

находим верхнюю оценку первого сомножителя в правой части (72):

$$
\sum_{n=1}^{M} \frac{\left\|\Delta_{n}^{*}\right\|_{4}^{4}}{\left\|\Delta_{n}^{*}\right\|_{2}^{2}} \leqslant C\left(A_{3}, A_{4}\right) \sum_{n=1}^{M} \sum_{\nu \in E_{2 n}}\left|c_{\nu}\right|^{2} \leqslant C\left(A_{3}, A_{4}\right) \sum_{n=1}^{N}\left|c_{n}\right|^{2} .
$$


С другой стороны, снова используя соотношения (69) и (70), имеем

$$
\sum_{n=1}^{M}\left\|\Delta_{n}^{*}\right\|_{2}^{2} \geqslant C_{1}\left(A_{1}, A_{4}\right) \sum_{n=1}^{N}\left|c_{n}\right|^{2} .
$$

Таким образом, неравенство (71) при $m_{N}=2^{2 M}$ следует из (72), (75), (76).

(II) Утверждение пункта (II) выводится из оценки (71) и того факта, что для любой функции $f \in H^{1}$ средние Валле Пуссена $\sigma_{n}(f)$ сходятся к $f$ по норме пространства Харди (см. [18]). Теорема 7 доказана.

Важным классом обобщенно-лакунарных спектров являются степенные спектры с целыми показателями $\left\{n^{h}\right\}$ при $h \geqslant 3$. В этом случае для числа решений диофантова уравнения

$$
n_{1}^{h}+n_{2}^{h}=n_{3}^{h}+n_{4}^{h},
$$

$n_{1}, n_{2}, n_{3}, n_{4} \leqslant N$, имеются асимптотические формулы К. Хооли [29]-[31], из которых следует, что выполняется условие (68).

Действительно, если $r_{h}(s)$ - число представлений натурального $s$ в виде

$$
s=n_{1}^{h}+n_{2}^{h},
$$

где $n_{1}, n_{2}$ - целые неотрицательные числа, то, как установлено в [31], имеет место соотношение

$$
\sum_{s \leqslant x} r_{h}^{2}(s)=C(h) x^{2 / h}+O\left(x^{5 /(3 h)+\varepsilon}\right), \quad \varepsilon>0 .
$$

Применяя теорему 7 и используя (79), получим следующее утверждение (cM. (71)).

Tеорема 8. Пусть спектр $\left\{m_{n}\right\}$ есть $\left\{n^{h}\right\}, h=3,4, \ldots$, u пусть коэффиииенты $\left\{c_{n}\right\}$ удовлетворяют условию (70).

(I) Если $f \in H^{1}$, то при всех $N=1,2, \ldots$

$$
\left\|\sigma_{N^{h}}(f)\right\|_{1} \geqslant C\left(A_{4}, h\right)\left(\sum_{n=1}^{N}\left|c_{n}\right|^{2}\right)^{1 / 2} .
$$

(II) Если $\sum_{n=1}^{\infty}\left|c_{n}\right|^{2}=\infty$, то ряд

$$
\sum_{n=1}^{\infty} c_{n} e^{i n^{h} x}
$$

не является рядом Фуръе.

ЗАмечАниЕ 6. Возможно, что результаты теоремы 8 сохраняют силу для всех спектров $\left[n^{\alpha}\right], \alpha>2$.

ЗАмечание 7 . Уравнение (77) имеет при $h \geqslant 3$ меньше нетривиальных решений, чем тривиальных (см. [29], [31]). Существует предположение о том, что при $h \geqslant 5$ уравнение (77) не имеет нетривиальных решений. Эта старая (включающая в себя последнюю теорему Ферма) проблема диофантовых уравнений остается нерешенной (см. [32]). 


\section{5. Квадратичный спектр, обобщение и усиление результатов Харди и Литтлвуда о тэта-рядах}

Число решений уравнения (77) для классического квадратичного спектра дается асимптотической формулой С. Рамануджана (см. [33]-[35]):

$$
\sum_{s \leqslant x} r_{2}^{2}(s)=\frac{x}{4}(\log x+C)+O\left(x^{3 / 5+\varepsilon}\right), \quad \varepsilon>0,
$$

где функция $r_{2}(s)$ определена соотношением (78). Следовательно (см. (80)), для спектра $m_{n}=n^{2}, n=1,2, \ldots$, соотношение (32) выполняется с показателями $\beta=2, \gamma=1$.

В силу теорем 5 и 6 для квадратичного спектра устанавливаются следующие важные результаты (см. (32), (38), (67)).

Теорема 9. Если $f \in H^{1}$ и имеет место разложение

$$
f(z)=\sum_{n=1}^{\infty} c_{n} z^{n^{2}}, \quad|z|<1,
$$

где коэфбициенты $\left\{c_{n}\right\}$ удовлетворяют при некотором $N=2,3, \ldots$ условию

$$
\left|c_{n}\right| \asymp n^{\rho} \omega(n), \quad 1 \leqslant n \leqslant 6 N,
$$

то справедливы следующие неравенства:

1) если $\rho>-1 / 2$, то

$$
\left\|\sigma_{N^{2}}(f)\right\|_{1} \geqslant C(\rho) N^{\rho+1 / 2} \omega(N)(\log N)^{-1 / 2} ;
$$

2) если $\rho=-1 / 2$ и для $\omega(n)$ выполняется (37), то

$$
\left\|\sigma_{N^{2}}(f)\right\|_{1} \gg \sum_{n=2}^{N} \frac{\omega(n)}{n \log n} .
$$

СлЕДСТвИЕ 1. При любой расстановке знаков справедливы следующие оценки (см. (82), $N \geqslant 3)$ :

$$
\begin{aligned}
& \text { 1) } \int_{0}^{2 \pi}\left|\sum_{n=1}^{N}\left( \pm \frac{1}{\sqrt{n}} e^{i n^{2} x}\right)\right| d x \gg \log \log N \\
& \text { 2) } \quad \int_{0}^{2 \pi}\left|\sum_{n=2}^{N}\left( \pm \frac{1}{\sqrt{n} \log \log n} e^{i n^{2} x}\right)\right| d x \gg \log \log \log N .
\end{aligned}
$$

Ввиду того, что средние Валле Пуссена сходятся по норме пространства Харди, соотношения (81)-(83) дают новые необходимые условия на модули коэффициентов Фурье комплексных тригонометрических рядов по полным квадратам. 
ТЕОРема 10. Пусть коэфбиииенты $\left\{c_{n}\right\}$ удовлетворяют соотношению

$$
\left|c_{n}\right| \asymp n^{\rho} \omega(n), \quad n=1,2, \ldots
$$

Тогда рядъ

$$
\sum_{n=1}^{\infty} c_{n} e^{i n^{2} x}
$$

не являются рядами Фуръе, если:

1) $\rho>-1 / 2$,

2) $\rho=-1 / 2, \omega(n)$ удовлетворяет условию (37) $и$

$$
\sum_{n=2}^{\infty} \frac{\omega(n)}{n \log n}=\infty
$$

СлЕДСТВИЕ 2. При любой расстановке знаков рядъ

$$
\sum_{n=2}^{\infty}\left( \pm \frac{1}{\sqrt{n} \log \log n} e^{i n^{2} x}\right)
$$

не являются рядами Фуръе.

ЗАмЕчАниЕ 8. Следствие 2 усиливает и обобщает (см. (84)-(86)) результаты Г. Харди и Дж. Литтлвуда (см. [16], [17], [19]), которые в исследованиях по эллиптическим тэта-функциям и связанным с ними тригонометрическим рядам установили, что ряды

$$
\sum_{n=1}^{\infty} \frac{1}{\sqrt{n}} e^{i n^{2} x} \quad \text { и } \sum_{n=1}^{\infty} \frac{(-1)^{n}}{\sqrt{n}} e^{i n^{2} x}
$$

не суммируются почти всюду методом средних арифметических и, следовательно, не являются рядами Фурье.

\section{6. Степенные спектры \\ с нецелыми показателями, комплексный случай}

Рассмотрим теперь ряды со спектрами $\left[n^{\alpha}\right], 1 \leqslant \alpha \leqslant 3 / 2$. Если $m_{n}=\left[n^{\alpha}\right]$, то при $1<\alpha<3 / 2$ И. И. Пятецкий-Шапиро и при $1<\alpha \leqslant 3 / 2$ М. З. Гараев (см. [36]) доказали, что число решений $Q(N)$ диофантова уравнения

$$
\left[n_{1}^{\alpha}\right]+\left[n_{2}^{\alpha}\right]=\left[n_{3}^{\alpha}\right]+\left[n_{4}^{\alpha}\right], \quad 0 \leqslant n_{1}, n_{2}, n_{3}, n_{4} \leqslant N
$$

удовлетворяет неравенству

$$
Q(N) \ll N^{4-\alpha}
$$


Возможно, что оценка (88) справедлива и при $3 / 2<\alpha<2$, но в настоящее время это неизвестно. М. З. Гараев использовал для оценки числа решений уравнения (87) тригонометрические суммы. Одновременно автор [11], [13] разработал новый комбинаторный подход к уравнениям (29), (87), не использующий их диофантовость. Представляется вероятным, что комбинаторными методами можно получить и верхнюю оценку С. Рамануджана (см. (80)) в случае произвольного выпуклого спектра.

В работах [11], [13] доказана комбинаторная теорема о разбиении отрезка, которая дает, в частности, оценку (88) без предположения о диофантовости уравнения (87).

Пусть произвольный отрезок множества вещественных чисел $[a, b]$ разбит на $N$ частей точками $\left\{t_{i}\right\}_{i=0}^{N}$ :

$$
a=t_{0}<t_{1}<\cdots<t_{N}=b .
$$

Обозначим через $\Delta_{i j}$ отрезки, порожденные этим разбиением:

$$
\Delta_{i j}=\left[t_{i}, t_{j}\right], \quad 0 \leqslant i<j \leqslant N .
$$

Пусть $Q(N)$ - число различных пар, состоящих из отрезков, имеющих одинаковую длину, т. е.

$$
Q(N)=\operatorname{card}\left\{\left(\Delta_{i j}, \Delta_{q r}\right):\left|\Delta_{i j}\right|=\left|\Delta_{q r}\right|\right\} .
$$

Обозначим через $R(i, j)$ число правых сдвигов отрезка $\Delta_{i j}$, т. е.

$$
R(i, j)=\operatorname{card}\left\{(q, r): q \geqslant i,\left|\Delta_{q r}\right|=\left|\Delta_{i j}\right|\right\} .
$$

В работах [11], [13] установлено, что справедлива следующая теорема.

Теорема 11. Пусть для некоторого $0<\alpha<\infty$ число правых сдвигов каждого отрезка $\Delta_{i j}$ удовлетворяет условию

$$
R(i, j) \ll(j-i)^{\alpha} .
$$

Тогда выполняется неравенство

$$
Q(N) \ll N^{2+\alpha /(\alpha+1)} .
$$

ЗАмечАниЕ 9. Неравенство (90) является точным в предельных случаях, когда $\alpha=0$ и $\alpha=\infty$. При $\alpha=1$ из теоремы 11 следует (см. (89)-(91)) установленная С. В. Конягиным [37] оценка:

$$
Q(N) \ll N^{5 / 2},
$$

если последовательность $\left\{t_{i}\right\}$ строго выпукла.

Из (87), (88) следует, что условие (32) выполняется для спектра $\left[n^{\alpha}\right], 1 \leqslant$ $\alpha \leqslant 3 / 2$, с показателями $\beta=4-\alpha, \gamma=0$. Поэтому теоремы 5 и 6 дают в этом случае следующие результаты (см. (32), (35), (66)). 
Теорема 12. Пусть $f \in H^{1}, 1 \leqslant \alpha \leqslant 3 / 2$. Предположим, что коэффиииенты $\left\{c_{n}\right\}$ разложения

$$
f(z)=\sum_{n=1}^{\infty} c_{n} z^{\left[n^{\alpha}\right]}, \quad|z|<1,
$$

удовлетворяют при некотором $N$ соотношению

$$
\left|c_{n}\right| \asymp n^{\rho} \omega(n), \quad 1 \leqslant n \leqslant 9 N .
$$

Тогда справедливо неравенство

$$
\left\|\sigma_{\left[N^{\alpha}\right]}(f)\right\|_{1} \geqslant \begin{cases}C(\rho) N^{\rho+(\alpha-1) / 2} \omega(N) & \text { nрu } \rho>\frac{1}{2}(1-\alpha), \\ C \sum_{n=1}^{N} \frac{\omega(n)}{n} & \text { nрu } \rho=\frac{1}{2}(1-\alpha) .\end{cases}
$$

СлЕДСтвиЕ 3. Для всех $1 \leqslant \alpha \leqslant 3 / 2$ при любой расстановке знаков имеют место неравенства

$$
\begin{aligned}
& \text { 1) } \int_{0}^{2 \pi}\left|\sum_{n=1}^{N}\left( \pm n^{(1-\alpha) / 2} e^{i\left[n^{\alpha}\right] x}\right)\right| d x \gg \log N \\
& \text { 2) } \quad \int_{0}^{2 \pi}\left|\sum_{n=2}^{N}\left( \pm n^{(1-\alpha) / 2}(\log n)^{-1} e^{i\left[n^{\alpha}\right] x}\right)\right| d x \gg \log \log N .
\end{aligned}
$$

Теорема 13. Если последовательность коэфбициентов $\left\{c_{n}\right\}$ определяется соотношением

$$
\left|c_{n}\right| \asymp n^{\rho} \omega(n), \quad n=1,2, \ldots,
$$

то при любом $1 \leqslant \alpha \leqslant 3 / 2$ ряд

$$
\sum_{n=1}^{\infty} c_{n} e^{i\left[n^{\alpha}\right] x}
$$

не является рядом Фуръе при $\rho>(1-\alpha) / 2$ или если $\rho=(1-\alpha) / 2$ и расходится ряд

$$
\sum_{n=1}^{\infty} \frac{\omega(n)}{n}=\infty .
$$

СлЕДСТвиЕ 4. При всех $1 \leqslant \alpha \leqslant 3 / 2$ рядъ

$$
\sum_{n=2}^{\infty}\left( \pm n^{(1-\alpha) / 2}(\log n)^{-1} e^{i\left[n^{\alpha}\right] x}\right)
$$

не являются рядами Фуръе ни при какой расстановке знаков.

ЗАмечАниЕ 10. В случае линейного спектра, когда $\alpha=1$, теоремы 12 и 13 являются точными и дают те же результаты, что и неравенство Харди (см. $(92)-(96))$. 


\section{7. Мультипликативные неравенства для $L_{1}$-нормы вещественных тригонометрических рядов и полиномов}

В вещественном случае вместо характеризации пространства $H^{1}$, определенной соотношением (8), аналогом теоремы Литтлвуда-Пэли является ограниченность функции Литтлвуда-Пэли для ряда Валле Пуссена как оператора из $L_{1}$ в пространство Лоренца $L_{1, \infty}$. Полное и детальное доказательство этой теоремы можно найти в работе автора [7].

Теорема 14. Для любой $f \in L_{1}(0,2 \pi)$ выполняется неравенство

$$
\operatorname{mes}\left\{\left(\sum_{n=0}^{\infty} \Delta_{n}^{2}(f, x)\right)^{1 / 2}>y\right\} \ll \frac{1}{y}\|f\|_{1},
$$

где (см. (3))

$$
\Delta_{n}(f, x)=\frac{1}{\pi} \int_{0}^{2 \pi} f(u) Q_{n}(x-u) d u .
$$

ЗАмечАниЕ 11. Отметим, что классическая функция Литтлвуда-Пэли для $f \in L_{1}$ может обращаться в бесконечность почти всюду. Действительно, с помощью соответствующей модификации конструкции А. Н. Колмогорова [38] можно построить такую функцию $f \in L_{1}$, для которой почти всюду

$$
\varlimsup_{n \rightarrow \infty}\left(S_{2^{n+1}}(f, x)-S_{2^{n}}(f, x)\right)=\infty .
$$

Однако в комплексном случае соотношение (99) не может иметь места, так как для любой $f \in H^{1}$ подпоследовательность частичных сумм ряда Фурье $S_{2^{n}}(f, x)$ сходится почти всюду (см. [18; т. 2, гл. XV, теорема 5.11]).

Теорема 14, а также характеризация пространства ВМО (см. (6)) являются основой для получения мультипликативных оценок $L_{1}$-нормы вещественных тригонометрических рядов. Однако - в отличие от пространства Харди, где мультипликативные неравенства включают в себя мультипликатор $\left\{\mu_{n}\right\}$, в качестве которого может быть взята любая последовательность неотрицательных чисел, - для пространства $L_{1}$ неравенства с убывающим мультипликатором $\left\{\mu_{n}\right\}$ не могут иметь места (см. [13]). А поскольку в исследовании свойств рядов с различными спектрами применялись именно убывающие мультипликаторы (см. теоремы 5 и 6), то мультипликативные неравенства в вещественном случае доказываются без дополнительного мультипликатора $\left\{\mu_{n}\right\}$.

Как и в комплексной ситуации (см. (9), (10)), будем применять разложение функции $f \in L_{1}(0,2 \pi)$ (которое сходится почти всюду)

$$
f(x)=\sum_{n=0}^{\infty} \Delta_{n}^{*}(f, x),
$$

использующее операторы

$$
\Delta_{n}^{*}(f, x)=\Delta_{2 n-1}(f, x)+\Delta_{2 n}(f, x),
$$

где $\Delta_{n}(f, x)$ определяется соотношением (98). 
Установим, что имеет место следующее мультипликативное неравенство, дающее нижнюю оценку $L_{1}$-нормы средних Валле Пуссена вещественных тригонометрических рядов Фурье.

Теорема 15. Пусть $f \in L_{1}(0,2 \pi)$. Тогда для каждого $2<p \leqslant \infty$ при всех $N=1,2, \ldots$ выполняется неравенство

$$
\left\|\sigma_{2^{2 N}}(f)\right\|_{1}\left(\sum_{n=0}^{N}\left(\frac{\left\|\Delta_{n}^{*}\right\|_{p}^{p}}{\left\|\Delta_{n}^{*}\right\|_{2}^{2}}\right)^{2 /(p-2)}\right)^{1 / 2} \geqslant C(p) \sum_{n=0}^{N}\left\|\Delta_{n}^{*}\right\|_{2}^{2} .
$$

ДоказАтельство. Пусть $2<p<\infty$. Положим (см. (98), (101))

$$
E_{n}=\left\{\left|\Delta_{n}^{*}(f, x)\right| \leqslant 2^{p /(2(p-2))}\left(\frac{\left\|\Delta_{n}^{*}\right\|_{p}^{p}}{\left\|\Delta_{n}^{*}\right\|_{2}^{2}}\right)^{1 /(p-2)}\right\} .
$$

Тогда справедливо следующее неравенство:

$$
\int_{C E_{n}}\left(\Delta_{n}^{*}(f, x)\right)^{2} d x \leqslant \pi\left\|\Delta_{n}^{*}\right\|_{2}^{2},
$$

где $C E_{n}=[0,2 \pi) \backslash E_{n}$.

Доказательство неравенства (104) аналогично доказательству соответствующего утверждения в комплексном случае (см. доказательство теоремы 3 , а именно соотношения (13)-(18)).

Введем функции $g_{n}(x), n=0,1, \ldots, N$ : положим (см. (103))

$$
g_{n}(x)= \begin{cases}\left|\Delta_{n}^{*}(f, x)\right| & \text { при } x \in E_{n}, \\ 0 & \text { при } x \in C E_{n} .\end{cases}
$$

В силу неравенства (104) имеем

$$
\int_{0}^{2 \pi} \sum_{n=0}^{N} g_{n}^{2}(x) d x \geqslant \pi \sum_{n=0}^{N}\left\|\Delta_{n}^{*}\right\|_{2}^{2}
$$

Оценим теперь левую часть этого неравенства сверху, используя теорему 14 . Введем множества $G_{y}, 0<y<\infty$ :

$$
G_{y}=\left\{\sum_{n=0}^{N} g_{n}^{2}(x)>y\right\}
$$

Имеем (см. (107))

$$
\int_{0}^{2 \pi} \sum_{n=0}^{N} g_{n}^{2}(x) d x=\int_{0}^{\sum_{n=0}^{N}\left\|g_{n}\right\|_{\infty}^{2}} \operatorname{mes} G_{y} d y .
$$

Далее, из (105) и (107) следует, что

$$
\operatorname{mes} G_{y} \leqslant \operatorname{mes} F_{y}
$$


где множество $F_{y}$ определяется соотношением

$$
F_{y}=\left\{\sum_{n=0}^{N}\left(\Delta_{n}^{*}(f, x)\right)^{2}>y\right\}
$$

Применяя неравенство (97) из теоремы 14 и учитывая при этом, что (см. (98), (101))

$$
\sum_{n=0}^{N} \Delta_{n}^{*}(f, x)=\sigma_{2^{2 N}}(f, x)
$$

а также опять используя (101), получим (см. (108)-(110))

$$
\begin{aligned}
& \int_{0}^{2 \pi} \sum_{n=0}^{N} g_{n}^{2}(x) d x \leqslant \int_{n=0}^{\sum_{n=0}^{N}\left\|g_{n}\right\|_{\infty}^{2}} \operatorname{mes} F_{y} d y \\
& \ll\left\|\sigma_{2^{2 N}}(f)\right\|_{1} \int_{0}^{\sum_{n=0}^{N}\left\|g_{n}\right\|_{\infty}^{2}} \frac{d y}{\sqrt{y}} \\
& \ll\left\|\sigma_{2^{2 N}}(f)\right\|_{1}\left(\sum_{n=0}^{N}\left\|g_{n}\right\|_{\infty}^{2}\right)^{1 / 2} .
\end{aligned}
$$

Но согласно соотношениям (103) и $(105)$, определяющим множества $E_{n}$ и функции $g_{n}(x)$, имеем

$$
\sum_{n=0}^{N}\left\|g_{n}\right\|_{\infty}^{2} \leqslant 2^{p /(p-2)} \sum_{n=0}^{N}\left(\frac{\left\|\Delta_{n}^{*}\right\|_{p}^{p}}{\left\|\Delta_{n}^{*}\right\|_{2}^{2}}\right)^{2 /(p-2)}
$$

Соединяя теперь неравенства (106), (111) и (112), получим

$$
\left\|\sigma_{2^{2 N}}(f)\right\|_{1}\left(\sum_{n=0}^{N}\left(\frac{\left\|\Delta_{n}^{*}\right\|_{p}^{p}}{\left\|\Delta_{n}^{*}\right\|_{2}^{2}}\right)^{2 /(p-2)}\right)^{1 / 2} \gg 2^{p /(2(2-p))} \sum_{n=0}^{N}\left\|\Delta_{n}^{*}\right\|_{2}^{2} .
$$

Тем самым (см. (102), (113)) теорема 15 при $2<p<\infty$ доказана.

Установим, что при $p=\infty$ имеет место более сильное утверждение (использующее характеризацию пространства ВМО через разложение Валле Пуссена), из которого, в частности, выводится теорема 15. Для этого понадобится следующее мультипликативное неравенство (см., например, [39]), которое может быть полезно и в других случаях.

Пусть $Q^{m}$ - единичный куб вещественного $m$-мерного пространства $\mathbb{R}^{m}$, $I \subseteq Q^{m}$ - некоторый $m$-мерный куб и $x=\left(x_{1}, \ldots, x_{m}\right)$.

Норма функции $f \in L_{1}\left(Q^{m}\right)$ в пространстве $\mathrm{BMO}\left(Q^{m}\right)$ определяется следующим образом:

$$
\|f\|_{\mathrm{BMO}}=\left|\int_{Q^{m}} f(x) d x\right|+\sup _{I} \frac{1}{|I|} \int_{I}\left|f(x)-f_{I}\right| d x,
$$

где $f_{I}=\frac{1}{|I|} \int_{I} f(x) d x$. 
Tеорема 16. Пусть $1 \leqslant q<r<\infty$. Если $f \in \operatorname{BMO}\left(Q^{m}\right)$, mo

$$
\|f\|_{r}^{r} \leqslant C(r, m)\|f\|_{\mathrm{BMO}}^{r-q}\|f\|_{q}^{q}
$$

Применяя неравенство (114) в периодическом варианте (при $m=1, r=2$ ) и используя тригонометрическую характеризацию пространства ВМО с разложением (9), (10) (см. замечание 2), находим, что имеет место следующий результат.

TeOpema 17. Пусть $f \in \mathrm{BMO}$. Тогда

$$
\|f\|_{2}^{2} \ll\|f\|_{1} \sup _{I}\left(\frac{1}{|I|} \int_{I} \sum_{2^{-n} \leqslant|I|}\left(\Delta_{n}^{*}(f, x)\right)^{2} d x\right)^{1 / 2} .
$$

Поскольку выполняется неравенство

$$
\sup _{I}\left(\frac{1}{|I|} \int_{I} \sum_{2^{-n} \leqslant|I|}\left(\Delta_{n}^{*}(f, x)\right)^{2} d x\right)^{1 / 2} \ll\left(\sum_{n=0}^{\infty}\left\|\Delta_{n}^{*}\right\|_{\infty}^{2}\right)^{1 / 2}
$$

то, применяя $(115)$ к $\sigma_{2^{2 n}}(f, x)$, получим теорему 15 для показателя $p=\infty$.

В работе [12] доказан также вариант теоремы 15 , использующий двоичные пачки Литтлвуда-Пэли (см. (2)).

TeOpema 18. Пусть $f \in L_{1}(0,2 \pi), 2<p \leqslant \infty$, u nусmъ

$$
\sum_{n=0}^{\infty}\left(\frac{\left\|\delta_{n}\right\|_{p}^{p}}{\left\|\delta_{n}\right\|_{2}^{2}}\right)^{2 /(p-2)}<\infty
$$

Тогда справедливо неравенство

$$
\|f\|_{1}\left(\sum_{n=0}^{\infty}\left(\frac{\left\|\delta_{n}\right\|_{p}^{p}}{\left\|\delta_{n}\right\|_{2}^{2}}\right)^{2 /(p-2)}\right)^{1 / 2} \geqslant C(p)\|f\|_{2}^{2} .
$$

ЗАмЕчАниЕ 12. В связи с классами функций, которые возникают в исследуемых в этой работе неравенствах, заметим, что, например, функционал $(p>r>0)$

$$
\left(\frac{\|f\|_{p}^{p}}{\|f\|_{r}^{r}}\right)^{1 /(p-r)}
$$

является "квазинормой" на конусе положительных функций, т. е. для него выполняется неравенство треугольника с константой, зависящей от $p$ и $r$, но, вообще говоря, (116) квазинормой не является. Отметим, что Беккенбах и Дрешер установили, что функционал (116) удовлетворяет неравенству треугольника при $p \geqslant 1 \geqslant r>0, f \geqslant 0$ (см. [40; гл. I, §23, 24]). 


\section{8. Вещественные тригонометрические ряды со спектрами степенной плотности}

Ввиду того, что неравенство (11) из теоремы 3 с убывающим мультипликатором теряет силу для вещественных тригонометрических рядов, соответствующие результаты здесь отличаются от тех, которые были установлены для пространства Харди. Вместе с тем для каждого из результатов, полученных для спектров степенной плотности в комплексном случае, имеется определенный вещественный аналог. Для $f \in L_{1}$ обозначим

$$
r_{n}=\left(a_{n}^{2}+b_{n}^{2}\right)^{1 / 2}, \quad n \geqslant 1,
$$

где $a_{n}, b_{n}$ - коэффициенты Фурье функции $f$.

ТеОрема 19. Пусть спектр $\left\{m_{n}\right\}$ имеет степенную плотность (см. (30)), а числа $R(q)$ (см. (29)) удовлетворяют соотношению (32), т.е. выполняется неравенство

$$
R(q) \ll \lambda_{q}^{\beta}\left(\log \lambda_{q}\right)^{\gamma},
$$

где $2 \leqslant \beta \leqslant 3, \gamma \leqslant 0$ при $\beta=3, \gamma \geqslant 0$ при $\beta=2$, и пусть функиия $f \in L_{1}(0,2 \pi)$ имеет ряд Фуръе

$$
f(x) \sim \sum_{n=1}^{\infty} r_{n} \cos \left(m_{n} x+\alpha_{n}\right)
$$

(I) Если для некоторого $N=1,2, \ldots$

$$
r_{n} \asymp n^{\rho} \omega(n) \quad \text { npu } 1 \leqslant n \leqslant N\left(1+A_{1}\right)^{2},
$$

то средние Валле Пуссена ряда (118) удовлетворяют неравенству

$$
\left\|\sigma_{m_{N}}(f)\right\|_{1} \geqslant\left\{\begin{array}{r}
C\left(A_{1}, A_{2}, \beta, \gamma, \rho\right) N^{\rho+(3-\beta) / 2} \omega(N)(\log N)^{-\gamma / 2} \\
n p u \beta \geqslant 2, \rho>\frac{1}{2}(\beta-3) \\
C\left(A_{1}, A_{2}, \beta, \gamma\right) \omega(N)(\log N)^{-\gamma / 2} \\
\text { npu } \beta>2, \rho=\frac{1}{2}(\beta-3) .
\end{array}\right.
$$

(II) $\operatorname{Ecлu~} \beta=2, \gamma \geqslant 0 u$

$$
r_{n} \asymp \frac{\omega(n)}{\sqrt{n}} \quad \text { nрu } 1 \leqslant n \leqslant N\left(1+A_{1}\right)^{2},
$$

где $\omega(n)$ удовлетворяет соотношению

$$
\sum_{n=1}^{m}\left(\omega\left(2^{n}\right)\right)^{2} \ll m\left(\omega\left(2^{m}\right)\right)^{2}, \quad m=1,2, \ldots,
$$


то выполняется неравенство

$$
\left\|\sigma_{m_{N}}(f)\right\|_{1} \geqslant C\left(A_{1}, A_{2}, \gamma\right) \omega(N)(\log N)^{(1-\gamma) / 2} .
$$

ДокАзАТЕЛЬСтво. Не ограничивая общности, можно считать, что (см. (39))

$$
m_{N}=2^{2 M}
$$

при некотором $M=1,2, \ldots$. Если

$$
2^{2(M-1)}<m_{N}<2^{2 M}
$$

то, как и при доказательстве теоремы 5, вместо разложения (100), (101) следует использовать вещественный вариант разложения (40), (41).

Оценим $\left\|\Delta_{n}^{*}\right\|_{4}^{4}$ сверху. Согласно обобщенному неравенству Минковского для этого достаточно оценить $\left\|\delta_{2 n-1}\right\|_{4}^{4},\left\|\delta_{2 n}\right\|_{4}^{4},\left\|\delta_{2 n+1}\right\|_{4}^{4}$ (см. (50), (98), (100), (101)). Оценивая, например, $\left\|\delta_{2 n}\right\|_{4}^{4}$ и используя ортогональность тригонометрической системы $\left\{\cos \left(n x+\alpha_{n}\right)\right\}$, имеем (см. (118))

$$
\left\|\delta_{2 n}\right\|_{4}^{4}=\frac{1}{8} \sum_{\substack{m_{\nu_{1}} \pm m_{\nu_{2}} \pm m_{\nu_{3}} \pm m_{\nu_{4}}=0 \\ \nu_{1}, \nu_{2}, \nu_{3}, \nu_{4} \in E_{2 n}}} r_{\nu_{1}} r_{\nu_{2}} r_{\nu_{3}} r_{\nu_{4}} .
$$

Обозначим через $Q(2 n)$ полное число решений уравнений

$$
m_{\nu_{1}} \pm m_{\nu_{2}} \pm m_{\nu_{3}} \pm m_{\nu_{4}}=0, \quad \nu_{j} \in E_{2 n}, \quad j=1,2,3,4
$$

при всех расстановках знаков.

Учитывая (124), в силу соотношения (123) получаем

$$
\left\|\delta_{2 n}\right\|_{4}^{4} \leqslant \frac{1}{8} Q(2 n) \max _{\nu \in E_{2 n}} r_{\nu}^{4} .
$$

Пусть $R_{1}(2 n)$ - число решений уравнения

$$
m_{\nu_{1}}-m_{\nu_{2}}=m_{\nu_{3}}+m_{\nu_{4}}
$$

где $\nu_{j} \in E_{2 n}, j=1,2,3,4$.

Докажем, что (см. (29))

$$
R_{1}(2 n) \leqslant R(2 n)
$$

Действительно, пусть $k(s)$ обозначает число представлений натурального $s$ в виде

$$
m_{\nu_{1}}+m_{\nu_{2}}=s, \quad \nu_{1}, \nu_{2} \in E_{2 n}
$$

и пусть $k_{1}(s)$ - число представлений $s$ в виде

$$
m_{\nu_{1}}-m_{\nu_{2}}=s, \quad \nu_{1}, \nu_{2} \in E_{2 n}
$$


Тогда, применяя неравенство Коши, имеем (см. (126))

$$
R_{1}(2 n)=\sum_{s=1}^{\infty} k(s) k_{1}(s) \leqslant\left(\sum_{s=1}^{\infty} k^{2}(s)\right)^{1 / 2}\left(\sum_{s=1}^{\infty} k_{1}^{2}(s)\right)^{1 / 2}
$$

Отсюда, учитывая, что

$$
\sum_{s=1}^{\infty} k_{1}^{2}(s) \leqslant \sum_{s=1}^{\infty} k^{2}(s)
$$

получим неравенство (127). А так как (см. (29), (124), (126))

$$
Q(2 n)=4\left(R(2 n)+R_{1}(2 n)\right)
$$

то из (125) и (127) следует, что

$$
\left\|\delta_{2 n}\right\|_{4}^{4} \leqslant R(2 n) \max _{\nu \in E_{2 n}} r_{\nu}^{4}
$$

Теперь, используя (32), (119), получим (см. (128))

$$
\left\|\Delta_{n}^{*}\right\|_{4}^{4} \ll \lambda_{2 n}^{\beta}\left(\log \lambda_{2 n}\right)^{\gamma} \max _{\nu \in E_{2 n}}(\omega(\nu))^{4} \nu^{4 \rho} .
$$

Далее, согласно (31), (42), (43), (46)-(48) при $\nu \in E_{2 n}$ выполняются соотношения

$$
\begin{gathered}
\nu \asymp C\left(A_{1}, A_{2}\right) \lambda_{2 n}, \\
\omega(\nu) \asymp C\left(A_{1}, A_{2}\right) \omega\left(2^{2 n}\right) .
\end{gathered}
$$

Поэтому, учитывая также, что (см. (59))

$$
\log \lambda_{2 n} \asymp C\left(A_{1}, A_{2}\right) n,
$$

получаем оценку (см. (129))

$$
\left\|\Delta_{n}^{*}\right\|_{4}^{4} \leqslant C\left(A_{1}, A_{2}, \beta, \gamma, \rho\right) \lambda_{2 n}^{4 \rho+\beta} n^{\gamma}\left(\omega\left(2^{2 n}\right)\right)^{4} .
$$

С другой стороны, ввиду (98), (100), (101), применяя равенство Парсеваля и снова используя соотношения (46)-(48), имеем

$$
\left\|\Delta_{n}^{*}\right\|_{2}^{2} \geqslant \sum_{\nu \in E_{2 n}}(\omega(\nu))^{2} \nu^{2 \rho} \geqslant C\left(A_{1}, A_{2}, \rho\right) \lambda_{2 n}^{2 \rho+1}\left(\omega\left(2^{2 n}\right)\right)^{2} .
$$


Применяя теорему 15 и учитывая, что $m_{N}=2^{2 M}$, получим в силу соотношений (101), (130), (131) следующее неравенство:

$$
\left\|\sigma_{2^{2 M}}(f)\right\|_{1}\left(\sum_{n=1}^{M} \lambda_{2 n}^{2 \rho+\beta-1}\left(\omega\left(2^{2 n}\right)\right)^{2} n^{\gamma}\right)^{1 / 2} \geqslant C\left(A_{1}, A_{2}, \beta, \gamma, \rho\right) \sum_{n=1}^{M} \lambda_{2 n}^{2 \rho+1}\left(\omega\left(2^{2 n}\right)\right)^{2} .
$$

Докажем пункт (I) теоремы 19. Поскольку и при $\beta \geqslant 2, \rho>(\beta-3) / 2$, и при $\beta>2, \rho=(\beta-3) / 2$ выполняются соотношения (см. (120))

$$
2 \rho+1>0, \quad 2 \rho+\beta>1
$$

то из (31) и (42) выводятся оценки (см. (133), (44), (48))

$$
\begin{aligned}
\sum_{n=1}^{M} \lambda_{2 n}^{2 \rho+\beta-1}\left(\omega\left(2^{2 n}\right)\right)^{2} n^{\gamma} \leqslant & C\left(A_{1}, A_{2}, \beta, \gamma, \rho\right) \\
& \times N^{2 \rho+\beta-1}(\omega(N))^{2}(\log N)^{\gamma}, \\
\sum_{n=1}^{M} \lambda_{2 n}^{2 \rho+1}\left(\omega\left(2^{2 n}\right)\right)^{2} & \asymp C\left(A_{1}, A_{2}, \rho\right) N^{2 \rho+1}(\omega(N))^{2} .
\end{aligned}
$$

Таким образом, неравенство (120) следует из неравенств (132), (134), (135), и пункт (I) доказан.

(II) Осталось рассмотреть важный случай (см. (120)), когда $\beta=2, \rho=$ $(\beta-3) / 2=-1 / 2, \gamma \geqslant 0$. Соотношения (130) и (131) дают здесь оценку

$$
\sum_{n=1}^{M} \frac{\left\|\Delta_{n}^{*}\right\|_{4}^{4}}{\left\|\Delta_{n}^{*}\right\|_{2}^{2}} \leqslant C\left(A_{1}, A_{2}, \gamma\right) \sum_{n=1}^{M} n^{\gamma}\left(\omega\left(2^{2 n}\right)\right)^{2}
$$

откуда, используя (31) и применяя (121), получим

$$
\sum_{n=1}^{M} \frac{\left\|\Delta_{n}^{*}\right\|_{4}^{4}}{\left\|\Delta_{n}^{*}\right\|_{2}^{2}} \leqslant C\left(A_{1}, A_{2}, \gamma\right) M^{1+\gamma}\left(\omega\left(2^{2 M}\right)\right)^{2}
$$

С другой стороны, (31) и (131) дают в рассматриваемом случае следующую оценку:

$$
\sum_{n=1}^{M}\left\|\Delta_{n}^{*}\right\|_{2}^{2} \geqslant \sum_{M / 2 \leqslant n \leqslant M}\left(\omega\left(2^{2 n}\right)\right)^{2} \geqslant C\left(A_{1}, A_{2}, \gamma\right) M\left(\omega\left(2^{2 M}\right)\right)^{2}
$$


Теперь, в силу неравенства (102), из теоремы 15 следует ввиду (136), (137), что

$$
\left\|\sigma_{2^{2 M}}(f)\right\|_{1} \geqslant C\left(A_{1}, A_{2}, \gamma\right) M^{(1-\gamma) / 2} \omega\left(2^{2 M}\right),
$$

и, таким образом, учитывая соотношения (39), (44), (46)-(48), имеем

$$
\left\|\sigma_{m_{N}}(f)\right\|_{1} \geqslant C\left(A_{1}, A_{2}, \gamma\right) \omega(N)(\log N)^{(1-\gamma) / 2} .
$$

Тем самым (см. (122)) теорема 19 полностью доказана.

Установим на основе теоремы 19 необходимые условия на модули коэффициентов Фурье рядов со спектрами степенной плотности в вещественном случае.

ТЕОРема 20. Пусть арифметические свойства спектра степенной плотности $\left\{m_{n}\right\}$ определяются соотношением (32):

$$
R(q) \leqslant \lambda_{q}^{\beta}\left(\log \lambda_{q}\right)^{\gamma}, \quad q=1,2, \ldots,
$$

а коэффичиенты тригонометрического ряда

$$
\sum_{n=1}^{\infty} r_{n} \cos \left(m_{n} x+\alpha_{n}\right)
$$

удовлетворяют условию

$$
r_{n} \asymp n^{\rho} \omega(n), \quad n=1,2, \ldots
$$

Тогда ряд (138) не является рядом Фуръе в следующих случаях:

1) $\rho>(\beta-3) / 2, \quad 2 \leqslant \beta \leqslant 3$;

2) $\rho=(\beta-3) / 2, \quad 2<\beta \leqslant 3, \quad \omega(n) \gg(\log n)^{\gamma / 2}, \quad n=1,2, \ldots$;

3) $\beta=2, \quad \rho=-1 / 2, \quad \gamma \geqslant 0, \quad \omega(n) \gg(\log n)^{(\gamma-1) / 2}, \quad n=1,2, \ldots$,

где для $\omega(n)$ выполняется (121).

ДокАЗАТЕЛЬство. Если $\rho>(\beta-3) / 2$, то из теоремы 19 следует, ввиду соотношений (31), (120), что для ряда (138)

$$
\left\|\sigma_{m_{N}}\right\|_{1} \rightarrow \infty \quad \text { при } N \rightarrow \infty
$$

и, таким образом (см. [18]), ряд (138) не является рядом Фурье.

Рассмотрим теперь случаи, когда $2 \leqslant \beta \leqslant 3, \rho=(\beta-3) / 2$ (см. (140), (141)). Здесь нужно использовать теорему Р. Салема о преобразовании рядов Фурье (см. [41], а также [17; т. 1, гл. IV, § 11, примечания]). 
Пусть, например, $\beta=2, \rho=(\beta-3) / 2=-1 / 2, \gamma \geqslant 0$, и выполняется (141). Предположим, что ряд (138) является рядом Фурье. Тогда согласно теореме Салема существует такая вогнутая последовательность $\Omega(n) \uparrow \infty$, для которой ряд

$$
\sum_{n=1}^{\infty} r_{n} \Omega\left(m_{n}\right) \cos \left(m_{n} x+\alpha_{n}\right)
$$

также есть ряд Фурье некоторой функции $f \in L_{1}$.

Поскольку последовательность $\{1 / \Omega(n)\}$ выпукла и стремится к нулю, то найдется настолько медленно возрастающая последовательность $\mu(n) \rightarrow \infty$, что

$$
\frac{\mu(n)}{\Omega(n)} \rightarrow 0,
$$

последовательность $\mu(n) / \Omega(n)$ выпукла и, кроме того, для подпоследовательности $\mu\left(m_{n}\right)$ выполняется условие $(31)$, т. е.

$$
\mu\left(m_{n^{2}}\right) \ll \mu\left(m_{n}\right), \quad n=1,2, \ldots .
$$

Из (143) следует (см. [17; т. 1 , гл. V, теорема 1.5]), что существует функция $g \in L_{1}$, рядом Фурье которой является ряд

$$
g(x) \sim \sum_{n=1}^{\infty} \frac{\mu(n)}{\Omega(n)} \cos n x .
$$

Пусть $h=f * g-$ свертка функций $f$ и $g$ :

$$
h(x)=\frac{1}{2 \pi} \int_{0}^{2 \pi} f(x-t) g(t) d t .
$$

Тогда функция $h(x)$ принадлежит $L_{1}(0,2 \pi)$ и ее ряд Фурье имеет следующий вид (см. (142)-(145)):

$$
h(x) \sim \sum_{n=1}^{\infty} r_{n} \mu\left(m_{n}\right) \cos \left(m_{n} x+\alpha_{n}\right) .
$$

Заметим, что поскольку $\mu(n) \uparrow \infty$, то условие (121) для последовательности $\left\{\mu\left(m_{n}\right) \omega(n)\right\}$ тоже выполняется. Применяя к ряду (146) теорему 19 (теорема остается справедливой, если $\{\omega(n)\}$ заменить на последовательность $\left\{\mu\left(m_{n}\right) \omega(n)\right\}$, которая является произведением двух монотонных последовательностей, удовлетворяющих условию (31)), получим в силу неравенства (122), что для средних Валле Пуссена этого ряда, ввиду (141), справедлива оценка

$$
\left\|\sigma_{m_{N}}(h)\right\|_{1} \gg \mu\left(m_{N}\right) \omega(N)(\log N)^{(1-\gamma) / 2} \gg \mu\left(m_{N}\right) .
$$

Полученное противоречие (см. (146), (147)) доказывает теорему 20 в рассматриваемом случае. Если $2<\beta \leqslant 3, \rho=(\beta-3) / 2$, то доказательство проводится аналогично. Теорема 20 доказана. 


\section{9. Квадратичный спектр и степенные спектры с нецелыми показателями, вещественный случай}

Рассмотрим сначала вещественные тригонометрические ряды со спектрами $\left\{n^{h}\right\}, h=3,4, \ldots$. Отметим, что теорема 7 об обобщенно-лакунарных спектрах остается справедливой в вещественном случае, поскольку она основывается на неравенстве (72), не содержащем убывающего мультипликатора и имеющем вещественный аналог (см. (102)). Поэтому, применяя результаты К. Хооли [29]-[31] и используя оценки (125) и (127), получим, что имеет место следующая теорема.

ТеОРема 21. Пусть $h=3,4, \ldots$, и пусть коэбфициенты тригонометрического ряда

$$
\sum_{n=1}^{\infty} r_{n} \cos \left(n^{h} x+\alpha_{n}\right)
$$

для каждого $q=2,3, \ldots$ удовлетворяют условию

$$
r_{n_{1}} \ll r_{n_{2}}
$$

npu $n_{1}, n_{2} \in E_{q-1} \cup E_{q} \cup E_{q+1}$.

(I) Если ряд (148) есть ряд Фуръе функиии $f \in L_{1}$, то для любого $N=$ $1,2, \ldots$ выполняется неравенство

$$
\left\|\sigma_{N^{h}}(f)\right\|_{1} \geqslant C(h)\left(\sum_{n=1}^{N} r_{n}^{2}\right)^{1 / 2} .
$$

(II) Если

$$
\sum_{n=1}^{\infty} r_{n}^{2}=\infty
$$

то ряд (148) не является рядом Фуръе.

Как и в случае тригонометрических рядов степенного типа, представляется вероятным, что теорема 21 сохраняет силу и для спектров $\left[n^{\alpha}\right]$ с нецелыми показателями $2<\alpha<\infty$ (см. (149), (150)). Однако для показателей $1 \leqslant \alpha \leqslant 2$ свойства вещественных и комплексных тригонометрических рядов со спектрами $\left[n^{\alpha}\right]$ уже существенно отличаются.

Обратимся к классическому спектру, образованному полными квадратами. Поскольку совокупное число решений системы уравнений (124) допускает оценку

$$
Q(n) \leqslant 8 R(n)
$$

где $R(n)$ - число решений уравнения (29), а $R(n)$ в свою очередь в случае спектра $\left\{m_{n}\right\}=\left\{n^{2}\right\}$ оцениваются на основе асимптотической формулы С. Рамануджана (см. (80)), то теорема 19 дает в этом случае следующий результат $(\beta=2, \gamma=1)$. 
Теорема 22. Пусть функиия $f \in L_{1}$ раскладьвается в ряд Фуръе по квадратичным гармоникам:

$$
f(x) \sim \sum_{n=1}^{\infty} r_{n} \cos \left(n^{2} x+\alpha_{n}\right),
$$

где числа $r_{n} \geqslant 0$ удовлетворяют при некотором $N$ соотношению

$$
r_{n} \asymp n^{\rho} \omega(n), \quad 1 \leqslant n \leqslant 6 N .
$$

Тогда справедливы следующие ощенки.

1. Если $\rho>-1 / 2$, то

$$
\left\|\sigma_{N^{2}}(f)\right\|_{1} \geqslant C(\rho) N^{\rho+1 / 2} \omega(N)(\log N)^{-1 / 2} .
$$

2. Если $\rho=-1 / 2, \omega(n)$ не убъвает и выполняется (121), то

$$
\left\|\sigma_{N^{2}}(f)\right\|_{1} \gg \omega(N)
$$

Теорема 20 устанавливает, в частности, важное условие на коэффициенты вещественного ряда с квадратичным спектром, необходимое для того, чтобы этот ряд являлся рядом Фурье.

ТЕОрема 23. Если коэффиииенты тригонометрического ряда

$$
\sum_{n=1}^{\infty}\left(a_{n} \cos n^{2} x+b_{n} \sin n^{2} x\right)
$$

удовлетворяют соотношению $(n=1,2, \ldots)$

$$
\left(a_{n}^{2}+b_{n}^{2}\right)^{1 / 2} \asymp n^{\rho}, \quad \rho \geqslant-\frac{1}{2},
$$

то ряд (151) не является рядом Фуръе.

ЗАмечАниЕ 13. Теорема 23 обобщает (см. (151), (152)) результат Г. Харди и Дж. Литтлвуда (см. [15], [16]), установивших, что два ряда

$$
\sum_{n=1}^{\infty} n^{\rho} \cos n^{2} x \quad \text { и } \quad \sum_{n=1}^{\infty} n^{\rho} \sin n^{2} x
$$

не являются рядами Фурье, если $\rho \geqslant-1 / 2$.

Рассмотрим теперь вещественные тригонометрические ряды со спектрами $\left[n^{\alpha}\right], 1 \leqslant \alpha \leqslant 3 / 2$. Из оценки (88) следует, что числа $R(q)$ (см. $(29)$ ) удовлетворяют соотношению (32) с показателями $\beta=4-\alpha, \gamma=0$. Поэтому теорема 19 приводит в этом случае к следующим оценкам интегральной нормы средних Валле Пуссена. 
Теорема 24. Пусть функиия $f$ принадлежит $L_{1}$ и имеет при $1 \leqslant \alpha \leqslant 3 / 2$ следующий ряд Фуръе:

$$
f(x) \sim \sum_{n=1}^{\infty}\left(a_{n} \cos \left[n^{\alpha}\right] x+b_{n} \sin \left[n^{\alpha}\right] x\right),
$$

где коэффициенты $a_{n}, b_{n}$ при некотором $N$ удовлетворяют соотношению

$$
\left(a_{n}^{2}+b_{n}^{2}\right)^{1 / 2} \asymp n^{\rho} \omega(n), \quad 1 \leqslant n \leqslant 9 N .
$$

Тогда справедливы следующие неравенства.

1. Если $\rho>(1-\alpha) / 2$, mо

$$
\left\|\sigma_{\left[N^{\alpha}\right]}(f)\right\|_{1} \geqslant C(\rho) N^{\rho+(\alpha-1) / 2} \omega(N) .
$$

2. Если $\rho=(1-\alpha) / 2$ и $\omega(n)$ не убъвает, то

$$
\left\|\sigma_{\left[N^{\alpha}\right]}(f)\right\|_{1} \geqslant \omega(N) .
$$

Применяя теорему 20 к спектру $\left[n^{\alpha}\right], 1 \leqslant \alpha \leqslant 3 / 2$, получим следующее необходимое условие на модули коэффициентов Фурье вещественных тригонометрических рядов с этим спектром.

TeOpema 25. Рядъ $(1 \leqslant \alpha \leqslant 3 / 2)$

$$
\sum_{n=1}^{\infty}\left(a_{n} \cos \left[n^{\alpha}\right] x+b_{n} \sin \left[n^{\alpha}\right] x\right)
$$

коэффициенты которых удовлетворяют соотношению $(n=1,2, \ldots)$

$$
\left(a_{n}^{2}+b_{n}^{2}\right)^{1 / 2} \asymp n^{\rho} \quad \text { nрu } \rho \geqslant \frac{1}{2}(1-\alpha),
$$

не являются рядами Фуръе.

ЗАмечАниЕ 14. Как и соответствующие результаты в комплексном случае (см. теорему 13), теорема 25 точна при $\alpha=1$ (см. [17; гл. V, теорема 1.5]). При $\alpha=2$ теорема 25 переходит в теорему 23. Представляется вероятным, что этот результат сохраняет силу при $3 / 2<\alpha<2$. 


\section{Список литературы}

[1] С. В. Бочкарев, "О проблеме Зигмунда", Изв. АН СССР. Сер. матем., 37:3 (1973), 630-638; англ. пер.: S. V. Bochkarev, "On a problem of Zygmund", Math. USSR-Izv., 7:3 (1973), 629-637.

[2] С. В. Бочкарев, "Об абсолютной сходимости рядов Фурье по ограниченным системам", Матем. заметки, 15:3 (1974), 363-370; англ. пер.: S. V. Bochkarev, "Absolute convergence of Fourier series with respect to bounded systems", Math. Notes, 15:3 (1974), 207-211.

[3] С. В. Бочкарев, “Об абсолютной сходимости рядов Фурье по ограниченным полным ортонормированным системам функций”, Матем. сб., 93(135):2 (1974), 203-217; англ. пер.: S. V. Bochkarev, "On the absolute convergence of Fourier series in bounded complete orthonormal systems of functions", Math. USSR-Sb., 22:1 (1974), 201-216.

[4] С. В. Бочкарев, "Расходящийся на множестве положительной меры ряд Фурье для произвольной ограниченной ортонормированной системы", Матем. сб., 98(140):3(11) (1975), 436-449; англ. пер.: S. V. Bochkarev, "A Fourier series in an arbitrary bounded orthonormal system that diverges on a set of positive measure", Math. USSR-Sb., 27:3 (1975), 393-405.

[5] С.В. Бочкарев, “Логарифмический рост средних арифметических от функций Лебега ограниченных ортонормированных систем", Докл. АН CCCP, 223:1 (1975), 16-19; англ. пер.: S. V. Bočkarev, "Logarithmic growth of the arithmetic means of the Lebesgue functions of bounded orthonormal systems", Soviet Math. Dokl., 16:4 (1975), 799-802.

[6] С. В. Бочкарев, "Метод усреднений в теории ортогональных рядов и некоторые вопросы теории базисов", Тр. МИАН СССР, 146, Наука, М., 1978, 3-87; англ. пер.: S. V. Bochkarev, "A method of averaging in the theory of orthogonal series and some problems in the theory of bases", Proc. Steklov Inst. Math., 146:3 (1980), 1-92.

[7] С. В. Бочкарев, "Ряды Валле-Пуссена в пространствах ВМО, $L_{1}$ и $H^{1}(D)$ и мультипликативные неравенства", Тр. МИАН, 210 (1995), 41-64; англ. пер.: S. V. Bochkarev, "Vallée-Poussin series in the spaces BMO, $L_{1}$, and $H^{1}(D)$, and multiplier inequalities", Proc. Steklov Inst. Math., 210 (1995), 30-46.

[8] С. В. Бочкарев, "Метод оценки $L_{1}$-нормы экспоненциальной суммы на основе арифметических свойств спектра", Функциональные пространства, гармонический анализ, дифференииальные уравнения, Сборник статей. К 95-летию со дня рождения академика Сергея Михайловича Никольского, Тр. МИАН, 232, Наука, M., 2001, 94-101; англ. пер.: S. V. Bochkarev, "A method for estimating the $L_{1}$ norm of an exponential sum based on arithmetic properties of the spectrum", Proc. Steklov Inst. Math., 232:1 (2001), 88-95.

[9] С. В. Бочкарев, "Новый метод оценки интегральной нормы экспоненциальных сумм. Приложение к квадратичным суммам", Докл. PAH, 386:4 (2002), 443-445; англ. пер.: S. V. Bochkarev, "A new method for estimating the integral norm of exponential sums: application to quadratic sums", Dokl. Math., 66:2 (2002), 210-212.

[10] С.В. Бочкарев, "Мультипликативные неравенства для функций из пространства Харди $H^{1}$ и их применение к оценке экспоненциальных сумм", Функциональные пространства, приближения, дифференииальные уравнения, Сборник статей. К 70-летию со дня рождения члена-корреспондента РАН Олега Владимировича Бесова, Тр. МИАН, 243, Наука, М., 2003, 96-103; англ. пер.: S. V. Bochkarev, "Multiplicative inequalities for functions from the Hardy space $H^{1}$ 
and their application to the estimation of exponential sums", Proc. Steklov Inst. Math., 243:4 (2003), 89-97.

[11] С. В. Бочкарев, "Новые мультипликативные неравенства и оценки $L_{1}$-нормы тригонометрических рядов и полиномов", Докл. РАН, 404:6 (2005), 727-730; англ. пер.: S. V. Bochkarev, "New multiplicative inequalities and estimates for the $L_{1}$-norm of trigonometric series and polynomials", Dokl. Math., 72:2 (2005), 762-765.

[12] С. В. Бочкарев, "Новые неравенства в теории Литтлвуда-Пэли и оценки $L_{1}$-нормы тригонометрических рядов и полиномов", Исследования по теории функиий и дифференииальным уравнениям, Сборник статей. К 100-летию со дня рождения академика Сергея Михайловича Никольского, Тр. МИАН, 248, Наука, М., 2005, 64-73; англ. пер.: S. V. Bochkarev, "New inequalities in the Littlewood-Paley theory and estimates of the $L_{1}$ norm of trigonometric series and polynomials", Proc. Steklov Inst. Math., 248 (2005), 59-68.

[13] С. В. Бочкарев, "Мультипликативные неравенства для $L_{1}$-нормы, применения в анализе и теории чисел", Функциональные пространства, теория приближений, нелинейный анализ, Сборник статей, Тр. МИАН, 255, Наука, М., 2006, 55-70; англ. пер.: S. V. Bochkarev, "Multiplicative inequalities for the $L_{1}$ norm: Applications in analysis and number theory", Proc. Steklov Inst. Math., 255 (2006), 49-64.

[14] С. В. Бочкарев, “Средние Валле Пуссена рядов Фурье для квадратичного спектра и спектров степенной плотности”, Докл. РАН, 439:3 (2011), 298-303; англ. пер.: S. V. Bochkarev, "De la Vallée-Poussin means of Fourier series for a quadratic spectrum and power density spectra", Dokl. Math., 84:1 (2011), 485-490.

[15] С. В. Бочкарев, "Метод усреднений в теории ортогональных рядов", Proceedings of the International Congress of Mathematicians, v. 2 (Helsinki, 1978), Acad. Sci. Fennica, Helsinki, 1980, 599-604.

[16] G. Hardy, J. Littlewood, "Some problems of Diophantine approximation", Proceedings of the 5th International Congress of Mathematicians, Cambridge, 1912, 223-229.

[17] G. Hardy, J. Littlewood, "Some problems of Diophantine approximation. II. The trigonometrical series associated with the elliptic $\theta$-functions", Acta Math., 37:1 (1914), 193-238.

[18] А. Зигмунд, Тригонометрические ряды, т. 1, 2, Мир, М., 1965, 615 с., 537 с.; пер. с англ.: A. Zygmund, Trigonometric series, v. I, II, 2nd ed., Cambridge Univ. Press, New York, 1959, xii+383 pp., vii+354 pp.

[19] G. Hardy, J. Littlewood, "Theorems concerning Cesàro means of power series", Proc. London Math. Soc. (2), 36:1 (1934), 516-531.

[20] Ch. Fefferman, "Characterizations of bounded mean oscillation", Bull. Amer. Math. Soc., 77:4 (1971), 587-588.

[21] Дж. Гарнетт, Ограниченнъе аналитические функиии, Мир, М., 1984, 470 с.; пер. с англ.: J.В. Garnett, Bounded analytic functions, Pure Appl. Math., 96, Academic Press, Inc. [Harcourt Brace Jovanovich, Publishers], New York-London, 1981, xvi+467 pp.

[22] С.В. Бочкарев, "Построение базисов в конечномерных пространствах аналитических в круге функций", Докл. АН СССР, 264:6 (1982), 1295-1297; англ. пер.: S. V. Bochkarev, "Construction of basis in finite-dimensional spaces of analytic functions in the disk", Soviet Math. Dokl., 25 (1982), 859-860. 
[23] С. В. Бочкарев, "Построение полиномиальных базисов в конечномерных пространствах аналитических в круге функций", Recent trends in mathematics (Reinhardsbrunn, October 11-13, 1982), Teubner-Texte Math., 50, BSB B. G Teubner Verlagsgesellschaft, Leipzig, 1982, 38-46.

[24] С. В. Бочкарев, "Построение полиномиальных базисов в конечномерных пространствах аналитических в круге функций", Ортогональные ряды и приближение функиий, Сборник статей. Посвящается 100-летию со дня рождения академика Н. Н. Лузина, Тр. МИАН СССР, 164, 1983, 49-74; англ. пер.: S. V. Bochkarev, "Construction of polynomial bases in finite-dimensional spaces of functions analytic in the disk", Proc. Steklov Inst. Math., 164 (1985), 55-81.

[25] R. R. Coifman, G. Weiss, "Extensions of Hardy spaces and their use in analysis", Bull. Amer. Math. Soc., 83:4 (1977), 569-645.

[26] С. В. Конягин, "О проблеме Литтлвуда", Изв. АН СССР. Сер. матем., 45:2 (1981), 243-265; англ. пер.: S. V. Konyagin, "On a problem of Littlewood", Math. USSR-Izv., 18:2 (1982), 205-225.

[27] O. C. McGehee, L. Pigno, B. Smith, "Hardy's inequality and the $L^{1}$ norm of exponential sums", Ann. of Math. (2), 113:3 (1981), 613-618.

[28] Р. Эдвардс, Рядъ Фуръе в современном изложении, т. 2, Мир, М., 1985, 400 с.; пер. с англ.: R. E. Edwards, Fourier series. A modern introduction, v. 2, 2nd ed., Grad. Texts in Math., 85, Springer-Verlag, New York-Berlin, 1982, xi+369 pp.

[29] C. Hooley, "On the representations of a number as the sum of two cubes", Math. Z., 82:3 (1963), 259-266.

[30] C. Hooley, "On the representation of a number as the sum of two $h$-th powers", Math. Z., 84:2 (1964), 126-136.

[31] C. Hooley, "On another sieve method and the numbers that are a sum of two $h$-th powers", Proc. Lond. Math. Soc. (3), 43:1 (1981), 73-109.

[32] C. Hooley, "Some recent advances in analytical number theory", Proceedings of the International Congress of Mathematicians, v. 1 (Warsaw, 1983), PWN, Warsaw, 1984, 85-97.

[33] S. Ramanujan, "Some formulae in the analytic theory of numbers", Messenger Math., 45 (1916), 81-84.

[34] S. Ramanujan, Collected papers, 2nd ed., Chelsea, New York, 1962.

[35] B. M. Wilson, "Proofs of some formulae enunciated by Ramanujan", Proc. Lond. Math. Soc. (2), 21:1 (1923), 235-255.

[36] M. Z. Garaev, "Upper bounds for the number of solutions of a Diophantine equation", Trans. Amer. Math. Soc., 357:6 (2005), 2527-2534.

[37] С. В. Конягин, "Об оценке $L_{1}$-нормы одной экспоненциальной суммы”, Теория приближений функиий и операторов, Тез. докл. международ. конф., Екатеринбург, 2000, 88-89.

[38] А.Н. Колмогоров, "Ряд Фурье-Лебега, расходящийся почти всюду", Избр. трудъ. Математика. Механика, ред. С. М. Никольский, Наука, М., 1985, 8-11; пер. с фр.: A. N. Kolmogoroff, "Une série de Fourier-Lebesgue divergente presque partout", Fund. Math., 4 (1923), 324-328.

[39] С. В. Бочкарев, "Теорема Хаусдорфа-Юнга-Рисса в пространствах Лоренца и мультипликативные неравенства", Теория приближений. Гармонический анализ, Сборник статей, посвященный памяти профессора Сергея Борисовича Стечкина, Тр. МИАН, 219, Наука, М., 1997, 103-114; англ. пер.: S. V. Bochkarev, 
"Hausdorff-Young-Riesz theorem in Lorentz spaces and multiplicative inequalities", Proc. Steklov Inst. Math., 219:4 (1997), 96-107.

[40] Э. Беккенбах, Р. Беллман, Неравенства, Мир, М., 1965, 276 с.; пер. с англ.: E. F. Beckenbach, R. Bellman, Inequalities, Ergeb. Math. Grenzgeb. (N.F.), 30, Springer-Verlag, Berlin-Göttingen-Heidelberg, 1961, xii+198 pp.

[41] R. Salem, "Sur les transformations des séries de Fourier", Fund. Math., 33 (1945), 108-114.

\section{Сергей Викторович Бочкарев} (Sergei V. Bochkarev)

Математический институт им. В. А. Стеклова РАН

E-mail: bochkarev@mi.ras.ru
Поступила в редакцию

24.01 .2013 